\title{
A class of quasi-variable mesh methods based on off-step discretization for the numerical solution of fourth-order quasi-linear parabolic partial differential equations
}

\author{
Ranjan Kumar Mohanty ${ }^{1 *}$ and Deepti Kaur ${ }^{2}$
}

"Correspondence:
rmohanty@sau.ac.in
'Department of Applied
Mathematics, Faculty of
Mathematics and Computer
Science, South Asian University,
Akbar Bhawan, Chanakyapuri, New
Delhi 110021, India
Full list of author information is
available at the end of the article

"Correspondence:

rmohanty@sau.ac.in

Mathematics, Faculty of

Mathematics and Computer

Delhi 110021, India

available at the end of the article

\begin{abstract}
Numerical schemes based on off-step discretization are developed to solve two classes of fourth-order time-dependent partial differential equations subjected to appropriate initial and boundary conditions. The difference methods reported here are second-order accurate in time and second-order accurate in space and, for a nonuniform grid, second-order accurate in time and third-order accurate in space. In case of a uniform grid, the second scheme is of order two in time and four in space. The presented methods split the original problem to a coupled system of two second-order equations and involve only three spatial grid points of a compact stencil without discretizing the boundary conditions. The linear stability of the presented methods has been examined, and it is shown that the proposed two-level finite difference method is unconditionally stable for a linear model problem. The new developed methods are directly applicable to fourth-order parabolic partial differential equations with singular coefficients, which is the main highlight of our work. The methods are successfully tested on singular problems. The proposed method is applied to find numerical solutions of the Euler-Bernoulli beam equation and complex fourth-order nonlinear equations like the good Boussinesq equation. Comparison of the obtained results with those for some earlier known methods show the superiority of the present approach.
\end{abstract}

Keywords: Euler-Bernoulli beam equation; off-step nodal points; quasi-variable mesh; finite difference method; successive tangential partial derivatives; good Boussinesq equation 
where $\Omega=\{(x, t) \mid-\infty<a<x<b<\infty, t>0\}$, equipped with the following initial and boundary conditions:

$$
u(x, 0)=u_{0}(x), \quad u_{t}(x, 0)=u_{1}(x), \quad a \leq x \leq b,
$$

and

$$
\begin{aligned}
& u(a, t)=g_{0}(t), \quad u(b, t)=g_{1}(t), \quad t>0, \\
& u_{x x}(a, t)=h_{0}(t), \quad u_{x x}(b, t)=h_{1}(t), \quad t>0,
\end{aligned}
$$

where $f, u_{0}, u_{1}, g_{0}, g_{1}, h_{0}$, and $h_{1}$ are functions of sufficient smoothness with required high-order derivatives.

Fourth-order PDEs arise in various mathematical models of physical problems in science and engineering such as vibrations of a homogenous beam, propagation of shallow water waves, fluid dynamics, surface diffusion of thin solid films, and deformation of beams [15]. Jacob Bernoulli formulated the first consistent elasticity theory of thin beams, in which the curvature of an elastic beam at any point is proportional to the bending moment at that point. Based on his uncle's elasticity theory, Daniel Bernoulli derived a PDE representing the motion of a thin vibrating beam $[1,2]$. Then, Leonard Euler extended and applied Bernoulli's theory to the loaded beams [6]. The Euler-Bernoulli beam equation is a fourth-order PDE governing the undamped transverse vibrations of a homogenous beam, in which the support does not contribute to the strain energy of the system and is set up as follows [6]:

$$
\frac{\partial^{2}}{\partial x^{2}}\left(\sigma(x) \frac{\partial^{2} u}{\partial x^{2}}\right)+\mu(x) \frac{\partial^{2} u}{\partial t^{2}}=p(x, t), \quad(x, t) \in \Omega
$$

where $u(x, t)$ is the transverse displacement of each position of the beam, $\sigma(x)>0$ is the flexural rigidity, $\mu(x)>0$ is the linear mass density, $p(x, t)$ is the load per unit length, and $b-a$ is the length of the beam. The quantity $u_{x x}$ is the value of the bending moment of the beam. Equation (3) must be solved subject to the initial conditions (2a) and simply supported boundary conditions (2b)-(2c). The solution of the Euler-Bernoulli beam equation (3) is significant in various branches of engineering such as the construction of flexible structures, the layout of robotic designs, and so on (see [1, 2]). The other time-dependent fourth-order PDE studied in the paper is the second-order Benjamin-Ono equation $[5,7]$ of the form

$$
q \frac{\partial^{4} u}{\partial x^{4}}+r \frac{\partial^{2}\left(u^{2}\right)}{\partial x^{2}}+\frac{\partial^{2} u}{\partial t^{2}}=0, \quad(x, t) \in \Omega
$$

where the constant $q$ denotes the depth of the fluid, and $r$ is a nonzero constant controlling nonlinearity and the characteristic speed of the long waves. In this case, the solution $u(x, t)$ is the elevation of the free surface of the fluid. It is one of the most important nonlinear PDEs arising in the study of water waves and is used in the analysis of many other physical applications such as the percolation of water in the porous subsurface of a horizontal layer of material [7]. We also consider the good Boussinesq equation, which is similar to the Korteweg-de Vries equation and presents a balance between dispersion and nonlinearity 
leading to the existence of soliton solutions [8]. The general form of the good Boussinesq equation can be written as

$$
\frac{\partial^{2} u}{\partial t^{2}}=\frac{\partial^{2} u}{\partial x^{2}}+\frac{\partial^{2} u^{2}}{\partial x^{2}}-\frac{\partial^{4} u}{\partial x^{4}}, \quad(x, t) \in \Omega .
$$

It is one of the important models having numerous applications in several fields, for instance, ion-acoustic waves in plasma, magnetohydrodynamics waves in plasma, longitudinal dispersive waves in elastic rods, pressure waves in liquid-gas bubble mixtures, and so on (see $[8,9])$. It describes shallow water waves propagating in both directions and possesses a highly complicated mechanism of solitary wave interaction [10].

Another particular class of fourth-order nonlinear parabolic PDEs considered in this study is of the form

$$
\frac{\partial^{4} u}{\partial x^{4}}-2 \frac{\partial^{3} u}{\partial x^{2} \partial t}+\frac{\partial^{2} u}{\partial t^{2}}=g\left(x, t, u, u_{x}, u_{x x}-u_{t}, u_{x x x}-u_{x t}\right), \quad(x, t) \in \Omega,
$$

subject to the initial and boundary conditions (2a)-(2c) (see [11]).

Owing to their great importance and wide range of applications, the attention of many physicists and mathematicians has been attracted to the studies of such problems. The closed-form solutions to fourth-order PDEs are necessary to know the qualitative behavior of natural processes and physical phenomena. But most fourth-order time-dependent PDEs have no closed-form solutions except for certain particular types of linear or quasilinear equations. Therefore, construction of accurate numerical methods for finding approximate solutions to these equations are of great significance. Among the entire arsenal of numerical methods available to approximate a fourth-order PDE, such as the finite element method, spline collocation method, the finite difference method, is attractive because of its relative ease of implementation, flexibility, and accuracy in the solution values. Higher-order methods yield not only comparable accuracy but also require much coarser discretization with greater computational efficiency. Apart from this, the advantage of developing a compact scheme restricted to the patch of cells immediately surrounding any given grid point is its suitability to be used directly adjacent to the boundary without introducing any extra nodes outside the boundary of the domain. Higher-order difference approximations for one-space-dimensional nonlinear parabolic and hyperbolic differential equations were discussed in [12-20]. A meshless numerical solution of hyperbolic PDEs using an improved localized radial basis functions collocation method was proposed in [21]. Recently, a new high-order compact implicit variable mesh discretization for onespace-dimensional unsteady quasi-linear biharmonic problem was developed in [22].

Various explicit and implicit difference schemes for numerical solution of the EulerBernoulli equation by decomposing it into a system of second-order PDEs have been studied by Conte [23], Crandall [24], Evans [25], Fairweather and Gourley [26], and Collatz [27]. The three-level explicit method suggested by Collatz [27] is easy to implement but is very time consuming even for the most modest problems due to the stability restriction. Andrade and McKee [28] suggested high-accuracy alternating direction implicit methods for solving fourth-order parabolic equations with variable coefficients. Using a multiderivative method, Twizell and Khaliq [29] derived a stable difference scheme for fourth-order parabolic equations with constant coefficients. Evans and Yousif [30] presented an unconditionally stable second-order accurate finite difference scheme using the 
alternating group explicit method achieving a better accuracy level. Later, Khan et al. [31] reported a three-level difference method of accuracy $O\left(k^{2}+h^{4}\right)$ for numerical solution of the Euler-Bernoulli equation by using a sextic spline in space and finite difference discretization in time. Further, Caglar and Caglar [32] considered a family of B-spline methods to produce accurate numerical solution of the Euler-Bernoulli equation. Rashidinia and Mohammadi [33] developed an approximation for finding the numerical solution of differential equation (3) by replacing the time derivative by a finite difference approximation and the space derivative by sextic spline functions using off-step points to obtain three-level implicit methods of accuracies $O\left(k^{2}+h^{4}\right)$ and $O\left(k^{4}+h^{4}\right)$. Mittal and Jain [34] discussed two new methods for solving the Euler-Bernoulli equation using B-splines with redefined basis functions. Most recently, Mohammadi [35] proposed a sextic B-spline collocation scheme for numerical solution of fourth-order time-dependent PDEs subjected to fixed and cantilever boundary conditions. Lai and Ma [7] proposed a lattice Boltzmann model for the second-order Benjamin-Ono equation (4). Numerous numerical methods have been proposed for solving the good Boussinesq equation (5) (see [8-10]). Recently, Siddiqi and Arshed [36] developed a quintic B-spline collocation method for finding an approximate solution of the good Boussinesq equation.

The consideration of using off-step nodal points for discretization is motivated by the polar form of one space Laplacian operator $\nabla^{2} \equiv \partial^{2} / \partial r^{2}+(\alpha / r)(\partial / \partial r)$, which has a singular coefficient associated with the first-order derivative term. Using only three grid points at each time level, three-level compact difference methods of order two in time and four in space for the solution of differential equation (1) for uniform mesh were reported by Mohanty and Evans [37], but these methods fail at singular points, and a special technique was needed to solve singular problems. To this concern, in the present article, using the same number of grid points $(3+3+3)$ of a single compact cell, we have proposed two new off-step discretizations for the solution of the fourth-order quasi-linear PDE (1) having the foremost advantage that these are directly applicable to the singular problems without requiring any fictitious points. Recently, Mohanty and Kaur [11] proposed an implicit highorder two-level finite difference scheme for the solution of particular type of fourth-order equation (6). However, that scheme featured a major shortcoming that it is not directly applicable to the singular problems and requires a special treatment to handle singular points. In this paper, we have developed two new two-level unconditionally stable implicit methods using off-step nodal points for the solution of the differential equation (6). The proposed new methods are convenient to implement at singular points without requiring any modification, and we do not need to discretize the boundary conditions, which is a main attraction.

An outline of the paper is as follows: In Section 2, we formulate and derive three-level quasi-variable mesh difference methods using off-step points for the solution of quasilinear fourth-order PDE (1). In Section 3, we present and derive new quasi-variable mesh two-level off-step discretizations to solve the particular type of fourth-order PDE (6). Further, in Section 4, the stability analysis of the derived methods for linear model problems have been discussed. In Section 5, we apply the proposed methods to a linear fourth-order PDE in polar coordinates. In Section 6, the performance of the proposed methods is illustrated by numerical experiments done on a collection of test problems having physical significance including the highly nonlinear good Boussinesq equation. Some concluding remarks about this paper are given in Section 7. 


\section{Three-level quasi-variable mesh off-step discretization and derivation}

For simplicity, we first consider the fourth-order nonlinear parabolic PDE of the form

$$
A(x, t) \frac{\partial^{4} u}{\partial x^{4}}+\frac{\partial^{2} u}{\partial t^{2}}=f\left(x, t, u, u_{t}, u_{x}, u_{x x}, u_{x x x}\right), \quad(x, t) \in \Omega
$$

We introduce the new variable $v$ defined as

$$
v=\frac{\partial^{2} u}{\partial x^{2}}
$$

Then equation (7) is reduced into an equivalent form of two second-order differential equations:

$$
\begin{aligned}
& \frac{\partial^{2} u}{\partial x^{2}}=v, \quad(x, t) \in \Omega, \\
& A(x, t) \frac{\partial^{2} v}{\partial x^{2}}+\frac{\partial^{2} u}{\partial t^{2}}=f\left(x, t, u, v, u_{t}, u_{x}, v_{x}\right), \quad(x, t) \in \Omega .
\end{aligned}
$$

Since the value of $u$ and $u_{t}$ is prescribed at $t=0$, this implies that the values of all successive tangential partial derivatives $u_{x}, u_{x x}, \ldots$ of $u$ are known at $t=0$. Since $v(x, 0)=u_{x x}(x, 0)$, the value of $v$ is also known at $t=0$. Also, note that the values of $u$ and $v$ are given at $x=a$ and $x=b$.

The associated initial and boundary conditions with (8a)-(8b) are

$$
\begin{array}{ll}
u(x, 0)=u_{0}(x), & v(x, 0)=u_{0}^{\prime \prime}(x), \\
u_{t}(x, 0)=u_{1}(x), & a \leq x \leq b, \\
u(a, t)=g_{0}(t), & v(a, t)=h_{0}(t), \quad t>0, \\
u(b, t)=g_{1}(t), & v(b, t)=h_{1}(t), \quad t>0 .
\end{array}
$$

In order to obtain a numerical solution of above initial boundary value problem, we superimpose on the solution domain $\Omega$ a rectangular grid with spacing $h_{l}=x_{l}-x_{l-1}, l=$ $1(1) N+1$, in the $x$-direction such that $a=x_{0}<x_{1}<\cdots<x_{N}<x_{N+1}=b, N$ being a positive integer, and $k=t_{j+1}-t_{j}>0$ in time direction. Spatial grid points are defined by $x_{l}=x_{0}+$ $\sum_{i=1}^{l} h_{i}, l=1(1) N+1$, and time steps are given by $t_{j}=j k, j=0,1,2, \ldots, J$, where $J$ is a positive integer. The mesh ratio is denoted by $\eta_{l}=\left(h_{l+1} / h_{l}\right)>0, l=1(1) N$. The neighboring off-step points are defined as $x_{l+1 / 2}=x_{l}+\frac{\eta_{l} h_{l}}{2}$ and $x_{l-1 / 2}=x_{l}-\frac{h_{l}}{2}, l=1(1) N$. For $\eta_{l}=1$, it reduces to the uniform mesh case. Let $u_{l}^{j}, v_{l}^{j}$ denote approximate solution values of $u(x, t), v(x, t)$ at the grid point $\left(x_{l}, t_{j}\right)$, and $U_{l}^{j}, V_{l}^{j}$ be their exact solution values at the grid point $\left(x_{l}, t_{j}\right)$, respectively. For $E=A, A_{x}$, and $A_{x x}$, let the values $E\left(x_{l}, t_{j}\right)$ be denoted by $E_{l}^{j}$. For simplicity, we consider $\eta_{l}=\eta$ (a constant $\neq 1$ ), $l=1(1) N$. Such a mesh is called a quasivariable mesh.

At the grid point $\left(x_{l}, t_{j}\right)$, for $S=A, U$, and $V$, we denote

$$
S_{a b}=\frac{\partial^{a+b} S}{\partial x^{a} \partial t^{b}}, \quad a, b=0,1, \ldots
$$


Let

$$
L=\eta^{2}+\eta-1, \quad M=(1+\eta)\left(1+3 \eta+\eta^{2}\right), \quad N=\eta\left(1+\eta-\eta^{2}\right) .
$$

At the grid point $\left(x_{l}, t_{j}\right)$, let

$$
\begin{aligned}
& P_{1_{l}}=\frac{(\eta-1)}{3}-\frac{h_{l}}{18}\left(1+\eta+\eta^{2}\right) \frac{A_{x_{l}}^{j}}{A_{l}^{j}}, \\
& P_{2_{l}}=\frac{1-\eta+\eta^{2}}{12}, \\
& P_{l}=\eta^{2}+\eta-1-\frac{h_{l}}{3}\left(1+\eta+\eta^{2}\right) \frac{A_{x_{l}}^{j}}{A_{l}^{j}}, \\
& Q_{l}=(1+\eta)\left(1+3 \eta+\eta^{2}\right)+\frac{h_{l}}{3}\left(1-\eta^{2}\right)\left(1+\eta+\eta^{2}\right) \frac{A_{x_{l}}^{j}}{A_{l}^{j}} \\
& R_{l}=\eta\left(1+\eta-\eta^{2}\right)+\frac{h_{l}}{3} \eta^{2}\left(1+\eta+\eta^{2}\right) \frac{A_{x_{l}}^{j}}{A_{l}^{j}} \\
& P_{l}^{*}=\eta^{2}-\frac{h_{l}}{6}\left(1+\eta+\eta^{2}\right) \frac{A_{x_{l}}^{j}}{A_{l}^{j}}, \\
& Q_{l}^{*}=\frac{\eta(1+\eta)}{2}+\frac{h_{l}}{6}\left(1-\eta^{2}\right)\left(1+\eta+\eta^{2}\right) \frac{A_{x_{l}}^{j}}{A_{l}^{j}}, \\
& R_{l}^{*}=\eta+\frac{h_{l}}{6} \eta^{2}\left(1+\eta+\eta^{2}\right) \frac{A_{x_{l}}^{j}}{A_{l}^{j}} .
\end{aligned}
$$

We require the following approximations for deriving the high-accuracy quasi-variable mesh methods. For $r=0, \pm 1$, we denote:

$$
\begin{aligned}
& \bar{U}_{l+r}^{j}=\theta U_{l+r}^{j+1}+(1-2 \theta) U_{l+r}^{j}+\theta U_{l+r}^{j-1}, \quad 0<\theta<1, \\
& \bar{U}_{l \pm 1 / 2}^{j}=\left(\bar{U}_{l \pm 1}^{j}+\bar{U}_{l}^{j}\right) / 2 \text {, } \\
& \bar{U}_{t_{l+r}}^{j}=\left(U_{l+r}^{j+1}-U_{l+r}^{j-1}\right) / 2 k \text {, } \\
& \bar{U}_{t_{l \pm 1 / 2}}^{j}=\left(U_{l \pm 1}^{j+1}+U_{l}^{j+1}-U_{l \pm 1}^{j-1}-U_{l}^{j-1}\right) / 4 k, \\
& \bar{u}_{t t_{l+r}}^{j}=\left(U_{l+r}^{j+1}-2 U_{l+r}^{j}+U_{l+r}^{j-1}\right) / k^{2} \text {, } \\
& \bar{u}_{x_{l}}^{j}=\left(\bar{u}_{l+1}^{j}-\left(1-\eta^{2}\right) \bar{u}_{l}^{j}-\eta^{2} \bar{u}_{l-1}^{j}\right) /\left(\eta(1+\eta) h_{l}\right) \text {, } \\
& \bar{u}_{x_{l+1 / 2}}^{j}=\left(\bar{U}_{l+1}^{j}-\bar{U}_{l}^{j}\right) /\left(\eta h_{l}\right), \\
& \bar{U}_{x_{l-1 / 2}}^{j}=\left(\bar{U}_{l}^{j}-\bar{U}_{l-1}^{j}\right) / h_{l}, \\
& \bar{U}_{x x_{l}}^{j}=\frac{2}{\eta(1+\eta) h_{l}^{2}}\left(\bar{U}_{l+1}^{j}-(1+\eta) \bar{U}_{l}^{j}+\eta \bar{U}_{l-1}^{j}\right) .
\end{aligned}
$$


Similarly, approximations are defined for the solution variable $v(x, t)$ at the grid point $\left(x_{l}, t_{j}\right)$ by replacing $U$ with $V$ in these expressions. Next, we define

$$
\begin{aligned}
\bar{F}_{l}^{j}=f\left(x_{l}, t_{j}, \bar{U}_{l}^{j}, \bar{V}_{l}^{j}, \bar{u}_{t_{l}}^{j}, \bar{U}_{x_{l}}^{j}, \bar{V}_{x_{l}}^{j}\right), \\
\bar{F}_{l \pm 1 / 2}^{j}=f\left(x_{l \pm 1 / 2}, t_{j}, \bar{u}_{l \pm 1 / 2}^{j}, \bar{V}_{l \pm 1 / 2}^{j}, \bar{U}_{t_{l \pm 1 / 2}}^{j}, \bar{U}_{x_{l \pm 1 / 2}}^{j}, \bar{V}_{x_{l \pm 1 / 2}}^{j}\right), \\
\overline{\bar{U}}_{l}^{j}=\bar{U}_{l}^{j}-\frac{\left(1-\eta+\eta^{2}\right)}{4} h_{l}^{2} \bar{U}_{x x_{l}}^{j}, \\
\overline{\bar{V}}_{l}^{j}=\bar{V}_{l}^{j}-\frac{\left(1-\eta+\eta^{2}\right)}{4} h_{l}^{2} \bar{V}_{x x_{l}}^{j}, \\
\overline{\bar{U}}_{x_{l}}^{j}=\bar{U}_{x_{l}}^{j}-\frac{\left(1+\eta+\eta^{2}\right)}{12(1+\eta)} h_{l}\left(\bar{V}_{l+1}^{j}-\bar{V}_{l-1}^{j}\right), \\
\overline{\bar{U}}_{t_{l}}^{j}=\bar{U}_{t_{l}}^{j}-\frac{\left(1-\eta+\eta^{2}\right)}{2 \eta(1+\eta)}\left(\bar{U}_{t_{l+1}}^{j}-(1+\eta) \bar{U}_{t_{l}}^{j}+\eta \bar{U}_{t_{l-1}}^{j}\right), \\
\overline{\bar{V}}_{x_{l}}^{j}=\bar{V}_{x_{l}}^{j}-\frac{\left(1+\eta+\eta^{2}\right)}{6(1+\eta) A_{l}^{j}} h_{l}\left(\bar{F}_{l+1 / 2}^{j}-\bar{F}_{l-1 / 2}^{j}\right) \\
\quad+\frac{\left(1+\eta+\eta^{2}\right)}{12(1+\eta) A_{l}^{j}} h_{l}\left(\bar{U}_{t t_{l+1}}^{j}-\bar{U}_{t t_{l-1}}^{j}\right)+\frac{\left(1+\eta+\eta^{2}\right) A_{x_{l}}^{j}}{12 A_{l}^{j}} h_{l}^{2} \bar{V}_{x x_{l}}^{j} .
\end{aligned}
$$

Finally, we let

$$
\overline{\bar{F}}_{l}^{j}=f\left(x_{l}, t_{j}, \overline{\bar{u}}_{l}^{j}, \overline{\bar{V}}_{l}^{j}, \overline{\bar{U}}_{t l}^{j}, \overline{\vec{u}}_{x_{l}}^{j}, \overline{\bar{V}}_{x_{l}}^{j}\right) .
$$

Then, at each grid point $\left(x_{l}, t_{j}\right), l=1(1) N, j=1,2, \ldots$, the proposed differential equations (8a)-(8b) are discretized by finite difference methods of accuracies $O\left(k^{2}+h_{l}^{2}\right)$ and $O\left(k^{2}+\right.$ $\left.k^{2} h_{l}+h_{l}^{3}\right)$ given by

$$
\begin{aligned}
& \bar{U}_{l+1}^{j}-(1+\eta) \bar{U}_{l}^{j}+\eta \bar{U}_{l-1}^{j} \\
&= \frac{h_{l}^{2}}{6}\left[(\eta-1) \bar{V}_{l+1}^{j}+(1+\eta)\left(1+\eta+\eta^{2}\right) \bar{V}_{l}^{j}-\eta^{2}(\eta-1) \bar{V}_{l-1}^{j}\right]+O\left(k^{2} h_{l}^{3}+h_{l}^{4}\right), \\
&\left(A_{00}+\frac{(\eta-1)}{3} h_{l} A_{10}\right)\left(\bar{V}_{l+1}^{j}-(1+\eta) \bar{V}_{l}^{j}+\eta \bar{V}_{l-1}^{j}\right) \\
&=-\frac{h_{l}^{2}}{6}\left[(\eta-1) \bar{U}_{t t_{l+1}}^{j}+(1+\eta)\left(1+\eta+\eta^{2}\right) \bar{U}_{t t_{l}}^{j}-\eta^{2}(\eta-1) \bar{U}_{t t_{l-1}}^{j}\right] \\
& \quad+\frac{h_{l}^{2}}{3}\left[(\eta-1) \bar{F}_{l+1 / 2}^{j}+(1+\eta)\left(1-\frac{\eta}{2}+\eta^{2}\right) \bar{F}_{l}^{j}-\eta^{2}(\eta-1) \bar{F}_{l-1 / 2}^{j}\right] \\
& \quad+O\left(k^{2} h_{l}^{2}+k^{2} h_{l}^{3}+h_{l}^{4}\right), \quad \eta \neq 1
\end{aligned}
$$

and

$$
\begin{aligned}
& \bar{U}_{l+1}^{j}-(1+\eta) \bar{U}_{l}^{j}+\eta \bar{U}_{l-1}^{j}=\frac{h_{l}^{2}}{12}\left[L \bar{V}_{l+1}^{j}+M \bar{V}_{l}^{j}+N \bar{V}_{l-1}^{j}\right]+\bar{T}_{l}^{j(1)}, \\
& \left(A_{00}+h_{l} P_{1_{l}} A_{10}+h_{l}^{2} P_{2_{l}} A_{20}\right)\left(\bar{V}_{l+1}^{j}-(1+\eta) \bar{V}_{l}^{j}+\eta \bar{V}_{l-1}^{j}\right) \\
& \quad=\frac{h_{l}^{2}}{12}\left[-P_{l} \bar{U}_{t t_{l+1}}^{j}-Q_{l} \bar{u}_{t t_{l}}^{j}-R_{l} \bar{U}_{t t_{l-1}}^{j}\right]+\frac{h_{l}^{2}}{3}\left[P_{l}^{*} \bar{F}_{l+1 / 2}^{j}+Q_{l}^{*} \overline{\bar{F}}_{l}+R_{l}^{*} \bar{F}_{l-1 / 2}^{j}\right]+\bar{T}_{l}^{j(2)},
\end{aligned}
$$


respectively, where $\bar{T}_{l}^{j(1)}=O\left(k^{2} h_{l}^{3}+h_{l}^{5}\right), \bar{T}_{l}^{j(2)}=O\left(k^{2} h_{l}^{2}+k^{2} h_{l}^{3}+h_{l}^{5}\right)$ for arbitrary $\theta$, provided that $\eta \neq 1$.

The derivation of the numerical methods (15a)-(15b) is straightforward. So, we discuss in detail the derivation of the novel off-step discretization technique given by (16a)-(16b).

At the grid point $\left(x_{l}, t_{j}\right)$, we let

$$
\alpha_{l}^{j}=\frac{\partial f}{\partial U}, \quad \beta_{l}^{j}=\frac{\partial f}{\partial V}, \quad \gamma_{l}^{j}=\frac{\partial f}{\partial U_{x}}, \quad \delta_{l}^{j}=\frac{\partial f}{\partial V_{x}}, \quad \xi_{l}^{j}=\frac{\partial f}{\partial U_{t}} .
$$

The differential equations $(8 \mathrm{a})-(8 \mathrm{~b})$ at the grid point $\left(x_{l}, t_{j}\right)$ may be written as

$$
\begin{aligned}
& U_{20}=V_{00}, \\
& A_{00} V_{20}+U_{02}=f\left(x, t_{j}, U_{l}^{j}, V_{l}^{j}, U_{t_{l}}^{j}, U_{x_{l}}^{j}, V_{x_{l}}^{j}\right) \equiv F_{l}^{j} .
\end{aligned}
$$

Similarly,

$$
F_{l \pm 1 / 2}^{j}=f\left(x_{l}, t_{j}, U_{l \pm 1 / 2}^{j}, V_{l \pm 1 / 2}^{j}, U_{t_{l \pm 1 / 2}}^{j}, U_{x_{l \pm 1 / 2}}^{j}, V_{x_{l \pm 1 / 2}}^{j}\right) \text {. }
$$

By using the Taylor series expansion in $\bar{F}_{l \pm 1 / 2}^{j}$, we obtain

$$
\begin{aligned}
& \bar{F}_{l+1 / 2}^{j}=F_{l+1 / 2}^{j}+\frac{k^{2}}{6} T_{1}+\frac{\eta^{2} h_{l}^{2}}{24} T_{2}+O\left(k^{2} h_{l}+h_{l}^{3}\right), \\
& \bar{F}_{l-1 / 2}^{j}=F_{l-1 / 2}^{j}+\frac{k^{2}}{6} T_{1}+\frac{h_{l}^{2}}{24} T_{2}+O\left(k^{2} h_{l}+h_{l}^{3}\right),
\end{aligned}
$$

where

$$
\begin{aligned}
& T_{1}=6 \theta\left(U_{02} \alpha_{l}^{j}+V_{02} \beta_{l}^{j}+U_{12} \gamma_{l}^{j}+V_{12} \delta_{l}^{j}\right)+U_{03} \xi_{l}^{j}, \\
& T_{2}=3 U_{20} \alpha_{l}^{j}+3 V_{20} \beta_{l}^{j}+U_{30} \gamma_{l}^{j}+V_{30} \delta_{l}^{j}+3 U_{21} \xi_{l}^{j} .
\end{aligned}
$$

Next, we let

$$
\begin{aligned}
& \overline{\bar{U}}_{l}^{j}=\bar{u}_{l}^{j}+a_{1} h_{l}^{2} \bar{u}_{x x_{l},}^{j}, \\
& \overline{\bar{V}}_{l}^{j}=\bar{V}_{l}^{j}+b_{1} h_{l}^{2} \bar{V}_{x x_{l},}^{j} \\
& \overline{\bar{U}}_{x_{l}}^{j}=\bar{U}_{x_{l}}^{j}+c_{1} h_{l}\left(\bar{V}_{l+1}^{j}-\bar{V}_{l-1}^{j}\right), \\
& \overline{\bar{V}}_{x_{l}}^{j}=\bar{V}_{x_{l}}^{j}+d_{1} h_{l}\left(\bar{F}_{l+1 / 2}^{j}-\bar{F}_{l-1 / 2}^{j}\right)+d_{2} h_{l}\left(\bar{u}_{t t_{l+1}}^{j}-\bar{u}_{t t_{l-1}}^{j}\right)+d_{3} h_{l}^{2} \bar{V}_{x x_{l}}^{j}, \\
& \overline{\bar{U}}_{t_{l}}^{j}=\bar{u}_{t_{l}}^{j}+e_{1}\left(\bar{u}_{t_{l+1}}^{j}-(1+\eta) \bar{u}_{t_{l}}^{j}+\eta \bar{u}_{t_{l-1}}^{j}\right),
\end{aligned}
$$

where $a_{1}, b_{1}, c_{1}, d_{1}, d_{2}, d_{3}$, and $e_{1}$ are the parameters to be determined in such a way that the truncation error $\bar{T}_{l}^{j(2)}$ is of accuracy $O\left(k^{2} h_{l}^{2}+k^{2} h_{l}^{3}+h_{l}^{5}\right)$.

Using approximations (12a)-(12i), with the help of equations (19a)-(19b), from (20a)(20e) we obtain

$$
\overline{\bar{U}}_{l}^{j}=U_{l}^{j}+\theta k^{2} U_{02}+\frac{h_{l}^{2}}{6} T_{3}+O\left(k^{4}+h_{l}^{3}\right),
$$




$$
\begin{aligned}
& \overline{\bar{V}}_{l}^{j}=V_{l}^{j}+\theta k^{2} V_{02}+\frac{h_{l}^{2}}{6} T_{4}+O\left(k^{4}+h_{l}^{3}\right), \\
& \overline{\bar{U}}_{x_{l}}^{j}=U_{x_{l}}^{j}+\theta k^{2} U_{12}+\frac{h_{l}^{2}}{6} T_{5}+O\left(k^{2} h_{l}^{2}+h_{l}^{3}\right), \\
& \overline{\bar{V}}_{x_{l}}^{j}=V_{x_{l}}^{j}+\theta k^{2} V_{12}+\frac{h_{l}^{2}}{6} T_{6}+O\left(k^{2} h_{l}+h_{l}^{3}\right), \\
& \overline{\bar{U}}_{t_{l}}^{j}=U_{t_{l}}^{j}+\frac{k^{2}}{6} U_{03}+\frac{h_{l}^{2}}{6} T_{7}+O\left(k^{2} h_{l}^{2}+h_{l}^{3}\right),
\end{aligned}
$$

where

$$
\begin{aligned}
& T_{3}=6 a_{1} U_{20} \\
& T_{4}=6 b_{1} V_{20} \\
& T_{5}=\left[\eta+6 c_{1}(1+\eta)\right] U_{30} \\
& T_{6}=\left[\left(\eta+3 d_{1}(1+\eta) A_{00}\right) V_{30}+6(1+\eta)\left(\frac{d_{1}}{2}+d_{2}\right) U_{12}+\left(3 d_{1}(1+\eta) A_{10}+6 d_{3}\right) V_{20}\right] \\
& T_{7}=3 e_{1} \eta(1+\eta) U_{21} .
\end{aligned}
$$

Finally, invoking the Taylor expansion and using (21a)-(21e) in (14), we obtain

$$
\overline{\bar{F}}_{l}^{j}=F_{l}^{j}+\frac{k^{2}}{6} T_{1}+\frac{h_{l}^{2}}{6} T_{8}+O\left(k^{2} h_{l}+h_{l}^{3}\right),
$$

where $T_{8}=T_{3} \alpha_{l}^{j}+T_{4} \beta_{l}^{j}+T_{5} \gamma_{l}^{j}+T_{6} \delta_{l}^{j}+T_{7} \xi_{l}^{j}$.

Further, by Taylor's series expansion we may write

$$
\begin{aligned}
\bar{U}_{l+1}^{j}-(1+\eta) \bar{U}_{l}^{j}+\eta \bar{U}_{l-1}^{j}= & U_{l+1}^{j}-(1+\eta) U_{l}^{j}+\eta U_{l-1}^{j}+\theta \frac{\eta(1+\eta)}{2} h_{l}^{2} k^{2} U_{22} \\
& +O\left(k^{2} h_{l}^{3}+k^{4}\right), \quad \eta \neq 1
\end{aligned}
$$

and

$$
\begin{aligned}
\left(A_{00}+\right. & \left.h_{l} P_{1_{l}} A_{10}+h_{l}^{2} P_{2} A_{20}\right)\left[\bar{V}_{l+1}^{j}-(1+\eta) \bar{V}_{l}^{j}+\eta \bar{V}_{l-1}^{j}\right] \\
= & \left(A_{00}+h_{l} P_{1_{l}} A_{10}+h_{l}^{2} P_{2_{l}} A_{20}\right)\left[V_{l+1}^{j}-(1+\eta) V_{l}^{j}+\eta V_{l-1}^{j}\right] \\
& +\theta \frac{\eta(1+\eta)}{2} h_{l}^{2} k^{2} A_{00} V_{22}+O\left(k^{2} h_{l}^{3}+k^{4}\right), \quad \eta \neq 1 .
\end{aligned}
$$

Since $U_{22}=V_{02}$, using relation (23a) in (16a), by the help of Taylor series the local truncation error $\bar{T}_{l}^{j^{(1)}}$ associated with (16a) may be obtained as $\bar{T}_{l}^{j^{(1)}}=O\left(k^{2} h_{l}^{3}+h_{l}^{5}\right)$ for arbitrary $\theta$. In a similar manner, by the help of approximations (12a)-(12d), (19a)-(19b), (22), and (23b), from (16b) we obtain the local truncation error $\bar{T}_{l}^{j^{(2)}}$ associated with (16b) as

$$
\begin{aligned}
\bar{T}_{l}^{j^{(2)}}= & -\frac{h_{l}^{2} k^{2}}{12} \eta(1+\eta)\left[T_{1}-\frac{U_{04}}{2}-6 \theta A_{00} V_{22}\right] \\
& -\frac{h_{l}^{4}}{72} \eta(1+\eta)\left[\left(1-\eta+\eta^{2}\right) T_{2}+2 T_{8}\right]+O\left(k^{2} h_{l}^{3}+h_{l}^{5}\right) .
\end{aligned}
$$


We observe from (24) that for the proposed method (16b) to be of accuracy $O\left(k^{2}+k^{2} h_{l}+\right.$ $h_{l}^{3}$ ), the coefficient of $h_{l}^{4}$ in (24) must be zero, that is, if and only if

$$
\left(1-\eta+\eta^{2}\right) T_{2}+2 T_{8}=0
$$

Thus, equating the coefficients of each of $U_{20}, V_{20}, U_{21}, U_{30}, V_{30}$, and $U_{12}$ in (25) to zero, we obtain the values of the parameters

$$
\begin{aligned}
& a_{1}=b_{1}=-\frac{\left(1-\eta+\eta^{2}\right)}{4}, \quad c_{1}=-\frac{\left(1+\eta+\eta^{2}\right)}{12(1+\eta)}, \\
& d_{1}=-\frac{\left(1+\eta+\eta^{2}\right)}{6(1+\eta) A_{00}}, \quad d_{2}=\frac{\left(1+\eta+\eta^{2}\right)}{12(1+\eta) A_{00}} \\
& d_{3}=\frac{\left(1+\eta+\eta^{2}\right) A_{10}}{12 A_{00}}, \\
& e_{1}=-\frac{\left(1-\eta+\eta^{2}\right)}{2 \eta(1+\eta)}
\end{aligned}
$$

Hence, we conclude that for this set of parameters, $\bar{T}_{l}^{(2)}=O\left(k^{2} h_{l}^{2}+k^{2} h_{l}^{3}+h_{l}^{5}\right)$ and the difference method (16b) is of accuracy $O\left(k^{2}+k^{2} h_{l}+h_{l}^{3}\right)$ for arbitrary $\theta$.

For the quasi-linear differential equation (1), that is, when the coefficient $A=A(x, t, u, v)$, we need to modify our proposed difference methods (15a)-(15b) and (16a)-(16b). In this case, we make use of the following approximations in (15a)-(15b) and (16a)-(16b):

$$
\begin{aligned}
& A_{10}=\frac{\bar{A}_{l+1}^{j}-\left(1-\eta^{2}\right) \bar{A}_{l}^{j}-\eta^{2} \bar{A}_{l-1}^{j}}{\eta(1+\eta) h_{l}}, \\
& A_{20}=\frac{2\left(\bar{A}_{l+1}^{j}-(1+\eta) \bar{A}_{l}^{j}+\eta \bar{A}_{l-1}^{j}\right)}{\eta(1+\eta) h_{l}^{2}},
\end{aligned}
$$

where

$$
\begin{aligned}
& \bar{A}_{l}^{j}=A\left(x_{l}, t_{j}, \bar{U}_{l}^{j}, \bar{V}_{l}^{j}\right), \\
& \bar{A}_{l \pm 1}^{j}=A\left(x_{l \pm 1}, t_{j}, \bar{U}_{l \pm 1}^{j}, \bar{V}_{l \pm 1}^{j}\right) .
\end{aligned}
$$

Using approximations (26a)-(26d), the difference methods (15a)-(15b) and (16a)-(16b) retain their orders, and hence we obtain difference methods of orders $O\left(k^{2}+h_{l}^{2}\right)$ and $O\left(k^{2}+k^{2} h_{l}+h_{l}^{3}\right)$, respectively, for the numerical solution of the quasi-linear equation (1).

When $\eta=1$ (constant mesh case), that is, for $h_{l+1}=h_{l}=h$, the proposed methods (15a)(15b) and (16a)-(16b) for the solution of the differential equations (8a)-(8b) reduces to the following implicit difference methods of orders $O\left(k^{2}+h^{2}\right)$ and $O\left(k^{2}+h^{4}\right)$ :

$$
\begin{aligned}
& \delta_{x}^{2} \bar{u}_{l}^{j}=h^{2} \bar{V}_{l}^{j}+O\left(k^{2} h^{2}+h^{4}\right), \\
& A_{00} \delta_{x}^{2} \bar{V}_{l}^{j}+h^{2} \bar{u}_{t t_{l}}^{j}=h^{2} \bar{F}_{l}^{j}+O\left(k^{2} h^{2}+h^{4}\right), \quad l=1(1) N, j=1,2, \ldots
\end{aligned}
$$

and

$$
\delta_{x}^{2} \bar{u}_{l}^{j}=\frac{h^{2}}{12}\left[\bar{V}_{l+1}^{j}+10 \bar{V}_{l}^{j}+\bar{V}_{l-1}^{j}\right]+O\left(k^{2} h^{4}+h^{6}\right),
$$




$$
\begin{gathered}
{\left[A_{00}+\frac{h^{2}}{12}\left(A_{20}-\frac{2 A_{10}^{2}}{A_{00}}\right)\right] \delta_{x}^{2} \bar{V}_{l}^{j}+\frac{h^{2}}{12}\left[\left(1-\frac{h A_{10}}{A_{00}}\right) \bar{U}_{t t_{l+1}}^{j}+10 \bar{U}_{t t_{l}}^{j}+\left(1+\frac{h A_{10}}{A_{00}}\right) \bar{U}_{t t_{l-1}}^{j}\right]} \\
=\frac{h^{2}}{3}\left[\left(1-\frac{h A_{10}}{2 A_{00}}\right) \bar{F}_{l+1 / 2}^{j}+\overline{\bar{F}}_{l}^{j}+\left(1+\frac{h A_{10}}{2 A_{00}}\right) \bar{F}_{l-1 / 2}^{j}\right]+O\left(k^{2} h^{2}+k^{2} h^{4}+h^{6}\right), \\
\quad l=1(1) N, j=1,2, \ldots,
\end{gathered}
$$

respectively, for arbitrary $\theta$.

Note that for the constant mesh case, the difference method (28a)-(28b) is fourth-order accurate in space for a fixed value of the mesh ratio parameter $\lambda=k / h^{2}$.

\section{Two-level off-step discretization strategy and truncation error analysis}

In this section, we develop new quasi-variable mesh off-step finite difference methods for the differential equation (6) with initial and boundary conditions given by (2a)-(2c).

Let us introduce the new variable $v(x, t)=u_{x x}(x, t)-u_{t}(x, t)$. Then we may rewrite the given PDE (6) in a coupled manner as

$$
\begin{aligned}
& \frac{\partial^{2} u}{\partial x^{2}}=\frac{\partial u}{\partial t}+v, \quad(x, t) \in \Omega, \\
& \frac{\partial^{2} v}{\partial x^{2}}=\frac{\partial v}{\partial t}+g\left(x, t, u, v, u_{x}, v_{x}\right), \quad(x, t) \in \Omega .
\end{aligned}
$$

Note that the initial and Dirichlet boundary conditions are given by $u(x, 0)=u_{0}(x)$, $u(a, t)=g_{0}(t)$, and $u(b, t)=g_{1}(t)$. Since the grid lines are parallel to the coordinate axes, this implies that the values of their successive tangential derivatives are known on the boundary, that is, the values of $u_{x x}(x, 0)=u_{0}^{\prime \prime}(x), u_{t}(a, t)=g_{0}^{\prime}(t)$, and $u_{t}(b, t)=g_{1}^{\prime}(t)$ are known exactly on the boundary.

The initial and boundary conditions associated with (29a)-(29b) can be written as

$$
\begin{array}{ll}
u(x, 0)=u_{0}(x), \quad v(x, 0)=u_{0}^{\prime \prime}(x)-u_{1}(x), & a \leq x \leq b \\
u(a, t)=g_{0}(t), \quad v(a, t)=h_{0}(t)-g_{0}^{\prime}(t), & u(b, t)=g_{1}(t), \\
v(b, t)=h_{1}(t)-g_{1}^{\prime}(t), \quad t>0 . &
\end{array}
$$

Let

$$
\widehat{t}_{j}=t_{j}+\tau k
$$

where $0<\tau<1$ is a parameter to be suitably determined.

Our quasi-variable mesh numerical methods are described as follows. For $p=0, \pm 1$, let

$$
\begin{aligned}
& \widehat{U}_{l+p}^{j}=\tau U_{l+p}^{j+1}+(1-\tau) U_{l+p}^{j}, \\
& \widehat{U}_{l \pm 1 / 2}^{j}=\left(\widehat{U}_{l \pm 1}^{j}+\widehat{U}_{l}^{j}\right) / 2, \\
& \widehat{U}_{l_{l+p}}^{j}=\left(U_{l+p}^{j+1}-U_{l+p}^{j}\right) / k, \\
& \widehat{U}_{x_{l}}^{j}=\left(\widehat{U}_{l+1}^{j}-\left(1-\eta^{2}\right) \widehat{U}_{l}^{j}-\eta^{2} \widehat{U}_{l-1}^{j}\right) /\left(\eta(1+\eta) h_{l}\right), \\
& \widehat{U}_{x_{l+1 / 2}}^{j}=\left(\widehat{U}_{l+1}^{j}-\widehat{U}_{l}^{j}\right) /\left(\eta h_{l}\right),
\end{aligned}
$$




$$
\begin{aligned}
& \widehat{U}_{x_{l-1 / 2}}^{j}=\left(\widehat{U}_{l}^{j}-\widehat{U}_{l-1}^{j}\right) / h_{l}, \\
& \widehat{U}_{x x_{l}}^{j}=\frac{2}{\eta(1+\eta) h_{l}^{2}}\left(\widehat{U}_{l+1}^{j}-(1+\eta) \widehat{U}_{l}^{j}+\eta \widehat{U}_{l-1}^{j}\right) .
\end{aligned}
$$

Replacing $U$ by $V$ in these expressions, similar approximations are defined for the solution variable $v(x, t)$ at the grid point $\left(x_{l}, t_{j}\right)$. Using these approximations, we define

$$
\begin{aligned}
& \widehat{G}_{l}^{j}=g\left(x_{l}, \widehat{t}_{j}, \widehat{U}_{l}^{j}, \widehat{V}_{l}^{j}, \widehat{U}_{x_{l}}^{j}, \widehat{V}_{x_{l}}^{j}\right), \\
& \widehat{G}_{l \pm 1 / 2}^{j}=g\left(x_{l \pm 1 / 2}, \widehat{t}_{j}, \widehat{U}_{l \pm 1 / 2}^{j}, \widehat{V}_{l \pm 1 / 2}^{j}, \widehat{U}_{x_{l \pm 1 / 2}}^{j}, \widehat{V}_{x_{l \pm 1 / 2}}^{j}\right) .
\end{aligned}
$$

Let

$$
\begin{aligned}
& \widehat{U}_{l}^{j}=\widehat{U}_{l}^{j}-\frac{\left(1-\eta+\eta^{2}\right)}{4} h_{l}^{2} \widehat{U}_{x x_{l},}^{j,} \\
& \widehat{\widehat{V}}_{l}^{j}=\widehat{V}_{l}^{j}-\frac{\left(1-\eta+\eta^{2}\right)}{4} h_{l}^{2} \widehat{V}_{x x_{l},}^{j}, \\
& \widehat{\widehat{U}}_{x_{l}}^{j}=\widehat{U}_{x_{l}}^{j}-\frac{\left(1+\eta+\eta^{2}\right)}{12(1+\eta)} h_{l}\left(\widehat{V}_{l+1}^{j}-\widehat{V}_{l-1}^{j}\right), \\
& \widehat{\widehat{V}}_{x_{l}}^{j}=\widehat{V}_{x_{l}}^{j}-\frac{\left(1+\eta+\eta^{2}\right)}{6(1+\eta)} h_{l}\left(\widehat{G}_{l+1 / 2}^{j}-\widehat{G}_{l-1 / 2}^{j}\right)-\frac{\left(1+\eta+\eta^{2}\right)}{12(1+\eta)} h_{l}\left(\widehat{V}_{t_{l+1}}^{j}-\widehat{V}_{t_{l-1}}^{j}\right) .
\end{aligned}
$$

Finally, we define

$$
\widehat{\widehat{G}}_{l}^{j}=g\left(x_{l}, \widehat{t}_{j}, \widehat{\widehat{U}}_{l}^{j}, \widehat{\widehat{V}}_{l}^{j}, \widehat{\widehat{U}}_{x_{l}}^{j}, \widehat{\widehat{V}}_{x_{l}}^{j}\right) .
$$

Then, at each internal grid point $\left(x_{l}, t_{j}\right), l=1(1) N, j=0,1,2, \ldots$, the finite difference methods of orders $O\left(k^{2}+h_{l}^{2}\right)$ and $O\left(k^{2}+k h_{l}+h_{l}^{3}\right)$ for the differential equations (29a)-(29b) are given by

$$
\begin{aligned}
\widehat{U}_{l+1}^{j} & -(1+\eta) \widehat{U}_{l}^{j}+\eta \widehat{U}_{l-1}^{j} \\
= & \frac{h_{l}^{2}}{6}\left[(\eta-1)\left(\widehat{U}_{t_{l+1}}^{j}+\widehat{V}_{l+1}^{j}\right)+(1+\eta)\left(1+\eta+\eta^{2}\right)\left(\widehat{U}_{t_{l}}^{j}+\widehat{V}_{l}^{j}\right)-\eta^{2}(\eta-1)\left(\widehat{U}_{t_{l-1}}^{j}+\bar{V}_{l-1}^{j}\right)\right] \\
& +O\left(k^{2} h_{l}^{2}+k h_{l}^{3}+h_{l}^{4}\right), \\
\widehat{V}_{l+1}^{j}- & (1+\eta) \widehat{V}_{l}^{j}+\eta \widehat{V}_{l-1}^{j} \\
= & \frac{h_{l}^{2}}{6}\left[(\eta-1) \widehat{V}_{t_{l+1}}^{j}+(1+\eta)\left(1+\eta+\eta^{2}\right) \widehat{V}_{t_{l}}^{j}-\eta^{2}(\eta-1) \widehat{V}_{t_{l-1}}^{j}\right] \\
& +\frac{h_{l}^{2}}{3}\left[(\eta-1) \widehat{G}_{l+1 / 2}^{j}+(1+\eta)\left(1-\frac{\eta}{2}+\eta^{2}\right) \widehat{G}_{l}^{j}-\eta^{2}(\eta-1) \widehat{G}_{l-1 / 2}^{j}\right] \\
& +O\left(k^{2} h_{l}^{2}+k h_{l}^{3}+h_{l}^{4}\right), \quad \eta \neq 1
\end{aligned}
$$

and

$$
\begin{aligned}
& \widehat{U}_{l+1}^{j}-(1+\eta) \widehat{U}_{l}^{j}+\eta \widehat{U}_{l-1}^{j} \\
& \quad=\frac{h_{l}^{2}}{6}\left[L\left(\widehat{U}_{t_{l+1}}^{j}+\widehat{V}_{l+1}^{j}\right)+M\left(\widehat{U}_{t_{l}}^{j}+\widehat{V}_{l}^{j}\right)+N\left(\widehat{U}_{t_{l-1}}^{j}+\bar{V}_{l-1}^{j}\right)\right]+\widehat{T}_{l}^{j(1)},
\end{aligned}
$$




$$
\begin{aligned}
\widehat{V}_{l+1}^{j}-(1+\eta) \widehat{V}_{l}^{j}+\eta \widehat{V}_{l-1}^{j} & \\
= & \frac{h_{l}^{2}}{6}\left[L \widehat{V}_{t_{l+1}}^{j}+M \bar{V}_{t_{l}}^{j}+N \bar{V}_{t_{l-1}}^{j}\right]+\frac{\eta h_{l}^{2}}{3}\left[\eta \widehat{G}_{l+1 / 2}^{j}+\frac{(1+\eta)}{2} \widehat{\widehat{G}}_{l}^{j}+\widehat{G}_{l-1 / 2}^{j}\right] \\
& +\widehat{T}_{l}^{(2)}, \quad \eta \neq 1,
\end{aligned}
$$

respectively, for $\tau=1 / 2$, where $\widehat{T}_{l}^{(1)}=O\left(k^{2} h_{l}^{2}+k h_{l}^{3}+h_{l}^{5}\right)$ and $\widehat{T}_{l}^{(2)}=O\left(k^{2} h_{l}^{2}+k h_{l}^{3}+h_{l}^{5}\right)$, provided that $\eta \neq 1$.

We discuss in detail the derivation of quasi-variable mesh finite difference method (37a)(37b). In this section, at the grid point $\left(x_{l}, t_{j}\right)$, we denote

$$
E=\frac{\partial g}{\partial t}, \quad H=\frac{\partial g}{\partial U}, \quad I=\frac{\partial g}{\partial V}, \quad J=\frac{\partial g}{\partial U_{x}}, \quad K=\frac{\partial g}{\partial V_{x}} .
$$

The proposed differential equations $(29 a)-(29 b)$ at the grid point $\left(x_{l}, t_{j}\right)$ can be written as

$$
\begin{aligned}
& U_{20}=U_{01}+V_{00}, \\
& V_{20}=V_{01}+g\left(x_{l}, t_{j}, U_{l}^{j}, V_{l}^{j}, U_{x l}^{j}, V_{x l}^{j}\right) \equiv G_{l}^{j} \quad \text { (say). }
\end{aligned}
$$

In a similar manner,

$$
G_{l \pm 1 / 2}^{j}=g\left(x_{l}, t_{j}, U_{l \pm 1 / 2}^{j}, V_{l \pm 1 / 2}^{j}, U_{x_{l \pm 1 / 2}^{j}}^{j}, V_{x_{l \pm 1 / 2}}^{j}\right) .
$$

The following relations are obtained upon differentiating system (29a)-(29b) with respect to $t$ at the grid point $\left(x_{l}, t_{j}\right)$ :

$$
\begin{aligned}
& U_{21}=U_{02}+V_{01}, \\
& V_{21}=V_{02}+E+H U_{01}+I V_{01}+J U_{11}+K V_{11} .
\end{aligned}
$$

By the help of approximations (32a)-(32f), from (33b) we get

$$
\begin{aligned}
& \widehat{G}_{l+1 / 2}^{j}=G_{l+1 / 2}^{j}+\tau k S_{1}+\frac{\eta^{2} h_{l}^{2}}{24} S_{2}+O\left(k^{2}+k h_{l}+h_{l}^{3}\right), \\
& \widehat{G}_{l-1 / 2}^{j}=G_{l-1 / 2}^{j}+\tau k S_{1}+\frac{h_{l}^{2}}{24} S_{2}+O\left(k^{2}+k h_{l}+h_{l}^{3}\right),
\end{aligned}
$$

where

$$
\begin{aligned}
& S_{1}=E+U_{01} H+V_{01} I+U_{11} J+V_{11} K, \\
& S_{2}=3 U_{20} H+3 V_{20} I+U_{30} J+V_{30} K .
\end{aligned}
$$

Now, let

$$
\begin{aligned}
& \widehat{\widehat{U}}_{l}^{j}=\widehat{U}_{l}^{j}+p_{1} h_{l}^{2} \widehat{U}_{x x_{l}}^{j}, \\
& \widehat{V}_{l}^{j}=\widehat{V}_{l}^{j}+q_{1} h_{l}^{2} \widehat{V}_{x x_{l}}^{j},
\end{aligned}
$$




$$
\begin{aligned}
& \widehat{U}_{x_{l}}^{j}=\widehat{U}_{x_{l}}^{j}+r_{1} h_{l}\left(\widehat{V}_{l+1}^{j}-\widehat{V}_{l-1}^{j}\right), \\
& \widehat{V}_{x_{l}}^{j}=\widehat{V}_{x_{l}}^{j}+s_{1} h_{l}\left(\widehat{G}_{l+1 / 2}^{j}-\widehat{G}_{l-1 / 2}^{j}\right)+s_{2} h_{l}\left(\widehat{V}_{t_{l+1}}^{j}-\widehat{V}_{t_{l-1}}^{j}\right),
\end{aligned}
$$

where $p_{1}, q_{1}, r_{1}, s_{1}$, and $s_{2}$ are the parameters to be determined in such a manner that the truncation error $\widehat{T}_{l}^{j^{(2)}}$ is of order $O\left(k^{2} h_{l}^{2}+k h_{l}^{3}+h_{l}^{5}\right)$.

Using approximations (32a)-(32f) and (41a)-(41b), we obtain

$$
\begin{aligned}
& \widehat{\widehat{U}}_{l}^{j}=U_{l}^{j}+\tau k U_{01}+\frac{h_{l}^{2}}{6} S_{3}+O\left(k^{2}+h_{l}^{3}\right), \\
& \widehat{\widehat{V}}_{l}^{j}=V_{l}^{j}+\tau k V_{01}+\frac{h_{l}^{2}}{6} S_{4}+O\left(k^{2}+h_{l}^{3}\right), \\
& \widehat{\widehat{U}}_{x_{l}}^{j}=U_{x_{l}}^{j}+\tau k U_{11}+\frac{h_{l}^{2}}{6} S_{5}+O\left(k^{2}+k h_{l}+h_{l}^{3}\right), \\
& \widehat{\widehat{V}}_{x_{l}}^{j}=V_{x_{l}}^{j}+\tau k V_{11}+\frac{h_{l}^{2}}{6} S_{6}+O\left(k^{2}+k h_{l}+h_{l}^{3}\right),
\end{aligned}
$$

where

$$
\begin{aligned}
& S_{3}=6 p_{1} U_{20}, \\
& S_{4}=6 q_{1} V_{20}, \\
& S_{5}=\left[\eta+6 r_{1}(1+\eta)\right] U_{30}, \\
& S_{6}=\left[\left(\eta+3 s_{1}(1+\eta)\right) V_{30}+6(1+\eta)\left(-\frac{s_{1}}{2}+s_{2}\right) V_{11}\right] .
\end{aligned}
$$

Using (31) and (43a)-(43d) in (35), we obtain

$$
\widehat{G}_{l}^{j}=G_{l}^{j}+\tau k S_{1}+\frac{h_{l}^{2}}{6} S_{7}+O\left(k^{2}+k h_{l}+h_{l}^{3}\right)
$$

where $S_{7}=S_{3} H+S_{4} I+S_{5} J+S_{6} K$.

Using relation (40a) and Taylor series, the local truncation error $\widehat{T}_{l}^{j^{(1)}}$ associated with (37a) is obtained as

$$
\widehat{T}_{l}^{j^{(1)}}=k h_{l}^{2} \frac{\eta(1+\eta)}{2}\left(\tau-\frac{1}{2}\right) U_{02}+O\left(k^{2} h_{l}^{2}+k h_{l}^{3}+h_{l}^{5}\right)
$$

For the proposed difference method (37a) to be of order $O\left(k^{2}+k h_{l}+h_{l}^{3}\right)$, the coefficient of $k h_{l}^{2}$ in (45) must be zero; thus, we obtain $\tau=\frac{1}{2}$, and the local truncation error $\widehat{T}_{l}^{j(1)}$ reduces to $O\left(k^{2} h_{l}^{2}+k h_{l}^{3}+h_{l}^{5}\right)$.

With the use of approximations (32a)-(32g), (41a)-(41b), and (44) in (37b) and relation (40b), taking $\tau=\frac{1}{2}$, the local truncation error $\widehat{T}_{l}^{j^{(2)}}$ associated with (37b) may be obtained as

$$
\widehat{T}_{l}^{j^{(2)}}=-\frac{h_{l}^{4}}{72} \eta(1+\eta)\left[\left(1-\eta+\eta^{2}\right) S_{2}+2 S_{7}\right]+O\left(k^{2} h_{l}^{2}+k h_{l}^{3}+h_{l}^{5}\right) .
$$


Thus, for the proposed difference method (37b) to be of order $O\left(k^{2}+k h_{l}+h_{l}^{3}\right)$, we must have

$$
\left(1-\eta+\eta^{2}\right) S_{2}+2 S_{7}=0
$$

Substituting the values of $S_{2}$ and $S_{7}$ into (47) and equating to zero the coefficients of $U_{20}, V_{20}, U_{30}, V_{30}$, and $V_{11}$, we obtain the following values of the parameters:

$$
\begin{aligned}
& p_{1}=q_{1}=-\frac{\left(1-\eta+\eta^{2}\right)}{4}, \quad r_{1}=-\frac{\left(1+\eta+\eta^{2}\right)}{12(1+\eta)}, \\
& s_{1}=-\frac{\left(1+\eta+\eta^{2}\right)}{6(1+\eta)}, \quad s_{2}=-\frac{\left(1+\eta+\eta^{2}\right)}{12(1+\eta)} .
\end{aligned}
$$

With this set of values, the local truncation error $\widehat{T}_{l}^{(2)}$ reduces to $O\left(k^{2} h_{l}^{2}+k h_{l}^{3}+h_{l}^{5}\right)$.

For $\eta=1$ (constant mesh case), that is, for $h_{l+1}=h_{l}=h$, for $\tau=\frac{1}{2}$, the proposed methods (36a)-(36b) and (37a)-(37b) for the solution of differential equations (29a)-(29b) reduce to the following implicit difference methods of orders $O\left(k^{2}+h^{2}\right)$ and $O\left(k^{2}+h^{4}\right)$ :

$$
\begin{aligned}
& \delta_{x}^{2} \widehat{U}_{l}^{j}=h^{2}\left(\widehat{U}_{t_{l}}^{j}+\widehat{V}_{l}^{j}\right)+O\left(k^{2} h^{2}+h^{4}\right), \\
& \delta_{x}^{2} \widehat{V}_{l}^{j}=h^{2}\left(\widehat{V}_{t_{l}}^{j}+\widehat{G}_{l}^{j}\right)+O\left(k^{2} h^{2}+h^{4}\right), \quad l=1(1) N, j=0,1,2, \ldots
\end{aligned}
$$

and

$$
\begin{aligned}
\delta_{x}^{2} \widehat{U}_{l}^{j}= & \frac{h^{2}}{12}\left[\left(\widehat{U}_{t_{l+1}}^{j}+\widehat{V}_{l+1}^{j}\right)+10\left(\widehat{U}_{t_{l}}^{j}+\widehat{V}_{l}^{j}\right)+\left(\widehat{U}_{t_{l-1}}^{j}+\widehat{V}_{l-1}^{j}\right)\right] \\
& +O\left(k^{2} h^{2}+k h^{4}+h^{6}\right), \\
\delta_{x}^{2} \widehat{V}_{l}^{j}= & \frac{h^{2}}{12}\left[\widehat{V}_{t_{l+1}}^{j}+10 \widehat{V}_{t_{l}}^{j}+\widehat{V}_{t_{l-1}}^{j}\right]+\frac{h^{2}}{3}\left[\widehat{G}_{l+1 / 2}^{j}+\widehat{\widehat{G}}_{l}^{j}+\widehat{G}_{l-1 / 2}^{j}\right] \\
& +O\left(k^{2} h^{2}+k h^{4}+h^{6}\right), \quad l=1(1) N, j=0,1,2, \ldots,
\end{aligned}
$$

respectively.

\section{Stability analysis using characteristic equation}

Let us consider the singularly perturbed model equation

$$
\epsilon \frac{\partial^{4} u}{\partial x^{4}}+\frac{\partial^{2} u}{\partial t^{2}}=f(x, t), \quad(x, t) \in \Omega
$$

where $0<\epsilon \ll 1$ is a small parameter. The proposed difference method (28a)-(28b) of or$\operatorname{der} O\left(k^{2}+h^{4}\right)$ for the uniform mesh when applied to this equation results in the following scheme written in the matrix form:

$$
S y^{j+1}=(2 S+T) y^{j}-S y^{j-1}+w
$$

where

$$
S=\left[\begin{array}{ll}
S_{11} & S_{12} \\
S_{21} & S_{22}
\end{array}\right], \quad T=\left[\begin{array}{ll}
T_{11} & T_{12} \\
T_{21} & T_{22}
\end{array}\right], \quad y=\left[\begin{array}{l}
u \\
v
\end{array}\right], \quad w=\left[\begin{array}{l}
w_{1} \\
w_{2}
\end{array}\right]
$$


The matrices $S$ and $T$ are $2 N \times 2 N$ block tridiagonal, $y$ is the $2 N$-component solution vector, and $w$ denotes the $2 N$ component column vector of known boundary values and right-hand side function values of the block system (51). The submatrices for $S$ and $T$ are given by

$$
\begin{aligned}
& S_{11}=12 \theta[1,-2,1], \quad S_{12}=-h^{2} \theta[1,10,1], \\
& S_{21}=[1,10,1], \quad S_{22}=12 \lambda^{2} h^{2} \epsilon \theta[1,-2,1], \\
& T_{11}=-12[1,-2,1], \quad T_{12}=h^{2}[1,10,1], \\
& T_{21}=[0,0,0], \quad T_{22}=-12 \lambda^{2} h^{2} \epsilon[1,-2,1],
\end{aligned}
$$

where $[a, b, c]$ is the $N \times N$ tridiagonal matrix having eigenvalues $b+2 \sqrt{a c} \cos (2 \phi), \phi=$ $(s \pi) /(2(N+1)), s=1(1) N$, and $\lambda=k / h^{2}$ is the mesh ratio parameter for the uniform mesh (for $\eta=1$, that is, for $h_{l+1}=h_{l}=h$ ). Here, $u=\left(u_{1}, u_{2}, \ldots, u_{N}\right)^{T}$ and $v=\left(v_{1}, v_{2}, \ldots, v_{N}\right)^{T}$ are solution vectors.

The eigenvalues of $S_{11}, S_{12}, S_{21}$, and $S_{22}$ are given by $-48 \theta \sin ^{2} \phi,-h^{2} \theta\left(12-4 \sin ^{2} \phi\right), 12-$ $4 \sin ^{2} \phi$, and $-48 \lambda^{2} h^{2} \epsilon \theta \sin ^{2} \phi$, respectively. Further, the eigenvalues of $T_{11}, T_{12}, T_{21}$, and $T_{22}$ are given by $48 \sin ^{2} \phi, h^{2}\left(12-4 \sin ^{2} \phi\right), 0$, and $48 \lambda^{2} h^{2} \epsilon \sin ^{2} \phi$, respectively.

For discussing the stability of the differential equation (50), we consider the homogenous part of the difference scheme (51), which may be written as

$$
\begin{aligned}
& y^{j+1}=\left(2 I+S^{-1} T\right) y^{j}-I z^{j}, \\
& z^{j+1}=I y^{j}+0 z^{j} .
\end{aligned}
$$

We denote by $\varepsilon_{1}^{j}=y^{j}-Y^{j}$ and $\varepsilon_{2}^{j}=z^{j}-Z^{j}$ the error vectors at the $j$ th iterate (in the absence of round-off errors), where

$$
Y^{j+1}=\left[\begin{array}{l}
U \\
V
\end{array}\right]^{j+1}, \quad Z^{j+1}=Y^{j}=\left[\begin{array}{l}
U \\
V
\end{array}\right]^{j},
$$

$U$ and $V$ being exact solution vectors.

We may write the error equation as

$$
E^{j+1}=\left[\begin{array}{l}
\varepsilon_{1} \\
\varepsilon_{2}
\end{array}\right]^{j+1}=H E^{j}
$$

where the amplification matrix $H$ is given by

$$
H=\left[\begin{array}{cc}
2 I+S^{-1} T & -I \\
I & 0
\end{array}\right] .
$$

The characteristic root $\xi$ of the matrix $S$ satisfies the following characteristic equation:

$$
\operatorname{det}\left[\begin{array}{cc}
-48 \theta \sin ^{2} \phi-\xi & -h^{2} \theta\left(12-4 \sin ^{2} \phi\right) \\
12-4 \sin ^{2} \phi & -48 \lambda^{2} h^{2} \epsilon \theta \sin ^{2} \phi-\xi
\end{array}\right]=0,
$$


which on simplification gives

$$
\begin{aligned}
\xi_{1,2}= & -24\left(1+\lambda^{2} h^{2} \epsilon\right) \theta \sin ^{2} \phi \\
& \pm \sqrt{576 \theta^{2}\left(1-\lambda^{2} h^{2} \epsilon\right) \sin ^{4} \phi-16 h^{2} \theta\left(6 \sin ^{2} \phi-9-\sin ^{4} \phi\right)} .
\end{aligned}
$$

The characteristic root $\rho$ of the matrix $T$ satisfies the following characteristic equation:

$$
\operatorname{det}\left[\begin{array}{cc}
-48 \sin ^{2} \phi-\rho & h^{2}\left(12-4 \sin ^{2} \phi\right) \\
0 & 48 \lambda^{2} h^{2} \epsilon \sin ^{2} \phi-\rho
\end{array}\right]=0,
$$

which gives

$$
\rho_{1}=48 \sin ^{2} \phi \quad \text { and } \quad \rho_{2}=48 \lambda^{2} h^{2} \epsilon \sin ^{2} \phi .
$$

Let $v$ be the eigenvalue of $S^{-1} T$, where $\xi$ and $\rho$ are eigenvalues of $S$ and $T$ satisfying (53) and (54), respectively. If $\mu$ denotes the characteristic root of the amplification matrix $H$, then it satisfies the following characteristic equation:

$$
\operatorname{det}\left[\begin{array}{cc}
2+v-\mu & -1 \\
1 & -\mu
\end{array}\right]=0
$$

which gives

$$
\mu^{2}-2 W \mu+1=0
$$

where $W=1+\frac{v}{2}$. Hence, we conclude that the difference method (28a)-(28b) is stable if $|W| \leq 1$.

For stability of the particular fourth-order PDE, we consider the linear parabolic equation of the form

$$
\frac{\partial^{4} u}{\partial x^{4}}-2 \frac{\partial^{3} u}{\partial x^{2} \partial t}+\frac{\partial^{2} u}{\partial t^{2}}=g(x, t), \quad(x, t) \in \Omega .
$$

Applying the method (48a)-(48b) of order $O\left(k^{2}+h^{2}\right)$ for the uniform mesh to the differential equation (56), we obtain the matrix equation

$$
Q y^{j+1}=R y^{j}+l,
$$

where

$$
Q=\left[\begin{array}{cc}
Q_{1} & Q_{2} \\
0 & Q_{1}
\end{array}\right], \quad R=\left[\begin{array}{cc}
R_{1} & R_{2} \\
0 & R_{1}
\end{array}\right], \quad y=\left[\begin{array}{c}
u \\
v
\end{array}\right], \quad l=\left[\begin{array}{l}
l_{1} \\
l_{2}
\end{array}\right],
$$

$u, v$ are solution vectors, and the vectors $l_{1}, l_{2}$ consist of homogenous functions, initial and boundary values of the block system (57). The submatrices $Q_{1}, Q_{2}, R_{1}$, and $R_{2}$ are given by

$$
Q_{1}=[0,1,0]-\frac{\lambda}{2}[1,-2,1], \quad Q_{2}=\frac{k}{2}[0,1,0],
$$




$$
R_{1}=[0,1,0]+\frac{\lambda}{2}[1,-2,1], \quad R_{2}=\frac{-k}{2}[0,1,0]
$$

The eigenvalues of submatrices $Q_{1}, Q_{2}, R_{1}$, and $R_{2}$ are given by $1+2 \lambda \sin ^{2} \phi, \frac{k}{2}, 1-2 \lambda \sin ^{2} \phi$, and $\frac{-k}{2}$, respectively. Hence, the eigenvalues of the matrices $Q$ and $R$ for the difference method (48a)-(48b) are given by $1+2 \lambda \sin ^{2} \phi$ and $1-2 \lambda \sin ^{2} \phi$, respectively.

The amplification matrix of system (57) is given by $Q^{-1} R$. Since the matrices $Q^{-1}$ and $R$ commute each other, the eigenvalues $\psi$ of $Q^{-1} R$ are given by

$$
\psi=\frac{1-2 \lambda \sin ^{2} \phi}{1+2 \lambda \sin ^{2} \phi}
$$

Since $0 \leq \sin ^{2} \phi \leq 1$, from (58) it is easy to verify that $|\psi| \leq 1$ for all variable angles $\phi$ and $\lambda>0$. Hence the method (48a)-(48b) is unconditionally stable for the differential equation (56).

\section{Application of the proposed difference methods to a linear singular equation}

Let us consider a class of linear singular equations of the form

$$
\nabla^{4} u+\frac{\partial^{2} u}{\partial t^{2}} \equiv\left(\frac{\partial^{2}}{\partial r^{2}}+\frac{\alpha}{r} \frac{\partial}{\partial r}\right)^{2} u+\frac{\partial^{2} u}{\partial t^{2}}=f(r, t), \quad 0<r<1, t>0
$$

equipped with the initial and boundary conditions of the form (2a)-(2c). Equivalently, equation (59) can be written in a coupled form as

$$
\begin{aligned}
& \frac{\partial^{2} u}{\partial r^{2}}=v, \\
& \frac{\partial^{2} v}{\partial r^{2}}+\frac{\partial^{2} u}{\partial t^{2}}=B(r) \frac{\partial^{3} u}{\partial r^{3}}+C(r) \frac{\partial^{2} u}{\partial r^{2}}+D(r) \frac{\partial u}{\partial r}+f(r, t), \quad 0<r<1, t>0,
\end{aligned}
$$

where

$$
B(r)=\frac{-2 \alpha}{r}, \quad C(r)=\frac{\alpha(2-\alpha)}{r^{2}}, \quad D(r)=\frac{\alpha(\alpha-2)}{r^{3}} .
$$

For $\alpha=1$ and 2 ,

$$
\nabla^{2} \equiv \frac{\partial^{2}}{\partial r^{2}}+\frac{\alpha}{r} \frac{\partial}{\partial r}
$$

denotes the Laplacian operator in cylindrical and spherical coordinates, respectively, in one space dimension.

Applying the difference method (15a)-(15b) to the singular equation (59), we obtain the following difference scheme of accuracy $O\left(k^{2}+h_{l}^{2}\right)$ :

$$
\begin{aligned}
& \bar{u}_{l+1}^{j}-(1+\eta) \bar{u}_{l}^{j}+\eta \bar{u}_{l-1}^{j}=\frac{h_{l}^{2}}{6}\left[(\eta-1) \bar{v}_{l+1}^{j}+(1+\eta)\left(1+\eta+\eta^{2}\right) \bar{v}_{l}^{j}-\eta^{2}(\eta-1) \bar{v}_{l-1}^{j}\right], \\
& \bar{v}_{l+1}^{j}-(1+\eta) \bar{v}_{l}^{j}+\eta \bar{v}_{l-1}^{j}+\frac{h_{l}^{2}}{6}\left[(\eta-1) \bar{u}_{t t_{l+1}}^{j}+(1+\eta)\left(1+\eta+\eta^{2}\right) \bar{u}_{t t_{l}}^{j}-\eta^{2}(\eta-1) \bar{U}_{t t_{l-1}}^{j}\right] \\
& \quad=\frac{h_{l}^{2}}{3}\left[(\eta-1)\left(B_{l+\frac{1}{2}} \bar{v}_{l+\frac{1}{2}}^{j}+C_{l+1 / 2} \bar{v}_{l+\frac{1}{2}}^{j}+D_{l+\frac{1}{2}} \bar{u}_{l+\frac{1}{2}}^{j}+f_{l+\frac{1}{2}}^{j}\right)\right.
\end{aligned}
$$




$$
\begin{aligned}
& +(1+\eta)\left(1-\frac{\eta}{2}+\eta^{2}\right)\left(B_{l} \bar{v}_{r_{l}}^{j}+C_{l} \bar{v}_{l}^{j}+D_{l} \bar{u}_{r_{l}}^{j}+f_{l}^{j}\right) \\
& \left.-\eta^{2}(\eta-1)\left(B_{l-\frac{1}{2}} \bar{v}_{r_{l-\frac{1}{2}}^{j}}^{j}+C_{l-\frac{1}{2}} \bar{v}_{l-\frac{1}{2}}^{j}+D_{l-\frac{1}{2}} \bar{u}_{r_{l-\frac{1}{2}}^{j}}^{j}+f_{l-\frac{1}{2}}^{j}\right)\right]
\end{aligned}
$$

where, for $p=0, \pm 1 / 2, B_{l+p}=B\left(r_{l+p}\right), C_{l+p}=C\left(r_{l+p}\right), D_{l+p}=D\left(r_{l+p}\right)$, and $f_{l+p}^{j}=f\left(r_{l+p}, t_{j}\right)$.

Similarly, applying the difference method (16a)-(16b) to the singular equation (59), we obtain the following difference scheme of accuracy $O\left(k^{2}+k^{2} h_{l}+h_{l}^{3}\right)$ :

$$
\begin{aligned}
& \bar{u}_{l+1}^{j}-(1+\eta) \bar{u}_{l}^{j}+\eta \bar{u}_{l-1}^{j}=\frac{h_{l}^{2}}{12}\left[L \bar{v}_{l+1}^{j}+M \bar{v}_{l}^{j}+N \bar{v}_{l-1}^{j}\right] \\
& \bar{v}_{l+1}^{j}-(1+\eta) \bar{v}_{l}^{j}+\eta \bar{v}_{l-1}^{j}+\frac{h_{l}^{2}}{12}\left[P_{l} \bar{u}_{t t_{l+1}}^{j}+Q_{l} \bar{u}_{t t_{l}}^{j}+R_{l} \bar{u}_{t t_{l-1}}^{j}\right] \\
& =\frac{\eta h_{l}^{2}}{3}\left[\left(\eta+p_{0}\right)\left(B_{l+\frac{1}{2}} \bar{v}_{l+\frac{1}{2}}^{j}+C_{l+\frac{1}{2}} \bar{v}_{l+\frac{1}{2}}^{j}+D_{l+\frac{1}{2}} \bar{u}_{r_{l+\frac{1}{2}}}^{j}+f_{l+\frac{1}{2}}^{j}\right)\right. \\
& +\frac{(1+\eta)}{2}\left(B_{l} \bar{v}_{r_{l}}^{j}+C_{l} \bar{v}_{l}^{j}+D_{l} \bar{u}_{r_{l}}^{j}+f_{l}^{j}+q_{0}\left(\bar{u}_{t t_{l+1}}^{j}-\bar{u}_{t t_{l-1}}^{j}\right)+r_{0} \bar{v}_{r r_{l}}^{j}+s_{0}\left(\bar{v}_{l+1}^{j}-\bar{v}_{l-1}^{j}\right)\right) \\
& \left.+\left(1-p_{0}\right)\left(B_{l-\frac{1}{2}} \bar{v}_{l-\frac{1}{2}}^{j}+C_{l-\frac{1}{2}} \bar{v}_{l-\frac{1}{2}}^{j}+D_{l-\frac{1}{2}} \bar{u}_{r_{l-\frac{1}{2}}}^{j}+f_{l-\frac{1}{2}}^{j}\right)\right],
\end{aligned}
$$

where

$$
\begin{array}{ll}
p_{0}=-\frac{\left(1+\eta+\eta^{2}\right)}{12} B_{l} h l, & q_{0}=\frac{\left(1+\eta+\eta^{2}\right)}{12(1+\eta)} B_{l} h_{l}, \\
r_{0}=-\frac{\left(1-\eta+\eta^{2}\right)}{4} C_{l} h_{l}^{2}, & s_{0}=-\frac{\left(1+\eta+\eta^{2}\right)}{12(1+\eta)} D_{l} h_{l} .
\end{array}
$$

Note that the quasi-variable mesh difference schemes (61a)-(61b) and (62a)-(62b) for the solution of singular equation (59) do not have the terms involving $1 /\left(r_{l \pm 1}\right)$, so the singularity at $r=0$ is avoided, and thus these schemes can be very easily solved in the region $[0<r<1] \times[t>0]$ without any modification. The difference scheme of accuracy $O\left(k^{2}+h^{4}\right)$ developed by Mohanty and Evans [37] using three spatial grid points for the uniform mesh featured a major drawback: it is not directly applicable to the singular equation (59) since it contains the term $F_{l-1}$, so a singularity arises at $l=1$ since $r_{0}=0$ and requires a special treatment to deal with the singular points. However, this is not the case with our proposed schemes since $F_{l-\frac{1}{2}}$ appears instead of $F_{l-1}$, which is the major advantage of using off-step discretization.

\section{Computational results}

In order to test the accuracy of the proposed methods, we have solved a large variety of linear and nonlinear fourth-order parabolic problems. In each case, the exact solution is prescribed and the right-hand side functions, the initial and boundary conditions, are obtained using the exact solution as a test procedure. We have chosen $\theta=0.5$ in each case for computing the solution of PDE (1), and all the computations were performed using MATLAB. The matrices represented by the new formulas are block tridiagonal. The Gauss-Seidel iteration method has been used for solving linear coupled system of equations, whereas the Newton nonlinear iteration method has been applied to determine the 
solution of nonlinear equations (see $[38,39]$ ), and in each case, the iterations are terminated once the absolute error tolerance $10^{-12}$ is reached.

Note that the proposed difference methods (15a)-(15b) and (16a)-(16b) are three-level in time. The values of $u$ and $v$ are known from the initial conditions. To begin any computation, it is necessary to know the values of $u$ and $v$ of required accuracy at the first time level, that is, at $t=k$. Using the known values of $u$ and $u_{t}$ at $t=0$, we can determine all their successive tangential partial derivatives at $t=0$, that is, the values of

$$
\frac{\partial^{r} u_{l}^{0}}{\partial x^{r}}, \frac{\partial^{r+1} u_{l}^{0}}{\partial x^{r} \partial t}, \frac{\partial^{r} v_{l}^{0}}{\partial x^{r}}, \frac{\partial^{r+1} v_{l}^{0}}{\partial x^{r} \partial t}, \quad r=0,1, \ldots,
$$

are known at $t=0$.

We use the following approximations for $u$ and $v$ of accuracy $O\left(k^{2}\right)$ at $t=k$ :

$$
\begin{aligned}
& U_{l}^{1}=U_{l}^{0}+k U_{t_{l}}^{0}+\frac{k^{2}}{2} U_{t t_{l}}^{0}+O\left(k^{3}\right), \\
& V_{l}^{1}=V_{l}^{0}+k V_{t_{l}}^{0}+\frac{k^{2}}{2} V_{t t_{l}}^{0}+O\left(k^{3}\right) .
\end{aligned}
$$

The considered fourth-order quasi-linear PDE (1) may be written as

$$
\frac{\partial^{2} u}{\partial t^{2}}=-A\left(x, t, u, u_{x x}\right) \frac{\partial^{4} u}{\partial x^{4}}+f\left(x, t, u, u_{t}, u_{x}, u_{x x}, u_{x x x}\right), \quad(x, t) \in \Omega .
$$

Differentiating (65) twice successively with respect to $x$ and using the relation $v=u_{x x}$, we get

$$
\frac{\partial^{2} v}{\partial t^{2}}=\frac{\partial^{2}}{\partial x^{2}}\left[-A\left(x, t, u, u_{x x}\right) \frac{\partial^{4} u}{\partial x^{4}}+f\left(x, t, u, v, u_{t}, u_{x}, v_{x}\right)\right], \quad(x, t) \in \Omega
$$

Using the initial values and their successive tangential partial derivatives in (65) and (66), we can determine the values of $U_{t t_{l}}^{0}$ and $V_{t t_{l}}^{0}$. Finally, substituting these values into (63) and (64), respectively, we can compute the values of $u$ and $v$ of required accuracy at $t=k$.

Throughout our computation (wherever not specified), we have used the time step $k=$ $1.6 /(N+1)^{2}$ for finding the solution at $t=1$. Since

$$
\begin{aligned}
b-a & =x_{N+1}-x_{0}=\left(x_{N+1}-x_{N}\right)+\left(x_{N}-x_{N-1}\right)+\cdots+\left(x_{1}-x_{0}\right)=h_{N+1}+h_{N}+\cdots+h_{1} \\
& =h_{1}\left(1+\eta+\eta^{2}+\cdots+\eta^{N}\right),
\end{aligned}
$$

so the first mesh spacing in the $x$-direction is obtained as

$$
h_{1}=\frac{(b-a)(1-\eta)}{1-\eta^{N+1}}, \quad \eta \neq 1 .
$$

Thus, we can calculate $h_{1}$ using (67) and mesh lengths of the remaining subintervals in the $x$-direction are computed by using the relation $h_{l+1}=\eta h_{l}, l=1(1) N$.

Example 1 We consider the Euler-Bernoulli beam equation (3) in the following form [3235]:

$$
\frac{\partial^{4} u}{\partial x^{4}}+\frac{\partial^{2} u}{\partial t^{2}}=\left(\pi^{4}-1\right) \sin \pi x \cos t, \quad 0<x<1, t>0 .
$$


Table 1 The absolute errors in the displacement $u$ and the bending moment $u_{x x}$ for the Euler-Bernoulli beam equation (68) for Example 1

\begin{tabular}{|c|c|c|c|c|c|c|c|c|c|}
\hline Methods & Time & $N+1$ & $k$ & & $x=0.1$ & $x=0.2$ & $x=0.3$ & $x=0.4$ & $x=0.5$ \\
\hline \multirow{8}{*}{$\begin{array}{l}\text { Proposed } \\
\text { method } \\
(28 a)-(28 b)\end{array}$} & \multirow[t]{2}{*}{0.02} & \multirow[t]{2}{*}{20} & \multirow[t]{2}{*}{0.00125} & $u$ & $1.53(-08)$ & $2.91(-08)$ & $4.00(-08)$ & $4.70(-08)$ & $4.95(-08)$ \\
\hline & & & & $u_{x x}$ & $1.52(-07)$ & $2.90(-07)$ & $3.99(-07)$ & $4.69(-07)$ & $4.93(-07)$ \\
\hline & \multirow[t]{2}{*}{0.02} & \multirow[t]{2}{*}{40} & \multirow[t]{2}{*}{0.00125} & $u$ & $5.07(-09)$ & $9.64(-09)$ & $1.33(-08)$ & $1.56(-08)$ & $1.64(-08)$ \\
\hline & & & & $u_{x x}$ & $5.01(-08)$ & $9.53(-08)$ & $1.31(-07)$ & $1.54(-07)$ & $1.62(-07)$ \\
\hline & \multirow[t]{2}{*}{0.05} & \multirow[t]{2}{*}{20} & \multirow[t]{2}{*}{0.005} & $u$ & $4.79(-07)$ & $9.11(-07)$ & $1.25(-06)$ & $1.47(-06)$ & $1.55(-06)$ \\
\hline & & & & $u_{x x}$ & $1.08(-05)$ & $2.05(-05)$ & $2.82(-05)$ & $3.31(-05)$ & $3.48(-05)$ \\
\hline & \multirow[t]{2}{*}{0.05} & \multirow[t]{2}{*}{40} & \multirow[t]{2}{*}{0.005} & $u$ & $4.19(-07)$ & $7.96(-07)$ & $1.10(-06)$ & $1.29(-06)$ & $1.35(-06)$ \\
\hline & & & & $u_{x x}$ & $3.16(-06)$ & $6.02(-06)$ & $8.28(-06)$ & $9.74(-06)$ & $1.02(-05)$ \\
\hline \multirow[t]{4}{*}{ Mohammadi [35] } & 0.02 & 20 & 0.00125 & $u$ & $4.29(-07)$ & $2.51(-07)$ & $1.24(-07)$ & $1.38(-07)$ & $1.40(-07)$ \\
\hline & 0.02 & 40 & 0.00125 & $u$ & $8.54(-08)$ & $6.23(-08)$ & $4.91(-08)$ & $5.07(-08)$ & $5.12(-08)$ \\
\hline & 0.05 & 20 & 0.005 & u & $2.96(-06)$ & $1.77(-06)$ & $1.64(-06)$ & $2.28(-06)$ & $2.65(-07)$ \\
\hline & 0.05 & 40 & 0.005 & $u$ & $9.07(-07)$ & $7.84(-07)$ & $7.69(-07)$ & $8.27(-07)$ & $8.61(-08)$ \\
\hline \multirow{2}{*}{$\begin{array}{l}\text { Mittal and Jain } \\
\text { [34] }\end{array}$} & 0.02 & 181 & 0.005 & $u$ & $1.50(-07)$ & $2.90(-07)$ & $3.90(-07)$ & $4.60(-07)$ & $4.90(-07)$ \\
\hline & 0.05 & 181 & 0.005 & u & $1.10(-06)$ & $2.09(-06)$ & $2.88(-06)$ & $3.38(-06)$ & $3.56(-06)$ \\
\hline Rashidinia and & 0.02 & 20 & 0.00125 & $u$ & $4.47(-07)$ & $2.66(-07)$ & $1.39(-07)$ & $1.55(-07)$ & $1.57(-07)$ \\
\hline Mohammadi [33] & 0.05 & 20 & 0.005 & $u$ & $2.91(-06)$ & $1.73(-06)$ & $1.60(-06)$ & $2.23(-06)$ & $2.60(-07)$ \\
\hline Caglar and & 0.02 & 121 & 0.005 & $u$ & $4.80(-06)$ & $9.70(-06)$ & $1.40(-05)$ & $1.90(-05)$ & $2.40(-05)$ \\
\hline \multirow[t]{2}{*}{ Caglar [32] } & 0.02 & 191 & 0.005 & $u$ & $5.20(-06)$ & $2.10(-06)$ & $3.10(-06)$ & $4.20(-06)$ & $5.20(-06)$ \\
\hline & 0.02 & 521 & 0.005 & $u$ & $4.90(-07)$ & $9.90(-07)$ & $1.40(-06)$ & $1.90(-06)$ & $2.40(-06)$ \\
\hline
\end{tabular}

The exact solution of this problem is

$$
u(x, t)=\sin \pi x \cos t .
$$

We have solved this problem by the proposed method (28a)-(28b) with $h=0.05,0.025$ and $k=0.00125,0.005$. The absolute errors in the displacement $u$ and the bending moment $u_{x x}$ at particular points $x=0.1,0.2,0.3,0.4,0.5$ are computed and reported in Table 1 for different time levels $t=0.02$ and $t=0.05$ using 16 and 10 time steps, respectively. We have compared our results with the results in [32-35], and it is evident from Table 1 that the proposed method (28a)-(28b) provides relatively more accurate solutions in comparison to the other existing methods. Figure 1(a) and 1(b) give a comparison of the plots of the exact and numerical solutions with $h=0.025$ and $k=0.005$ for $t=0$ to 0.5 .

Example 2 We consider the following nonhomogenous fourth-order parabolic equation [33]:

$$
\frac{\partial^{2} u}{\partial t^{2}}+(1+x) \frac{\partial^{4} u}{\partial x^{4}}=\left(x^{3}+x^{4}-\frac{6}{7 !} x^{7}\right) \cos t, \quad 0<x<1, t>0 .
$$

The exact solution is

$$
u(x, t)=\frac{6}{7 !} x^{7} \cos t
$$

We have solved this problem using method (28a)-(28b) with $h=0.05$ and $k=0.00125$ using 16 time steps. The absolute errors in $u$ and $u_{x x}$ at particular points $x=0.1,0.2,0.3$, $0.4,0.5$ are tabulated in Table 2 at $t=0.02$ and compared with the results reported in [33]. These results verify the superiority of the proposed method. 


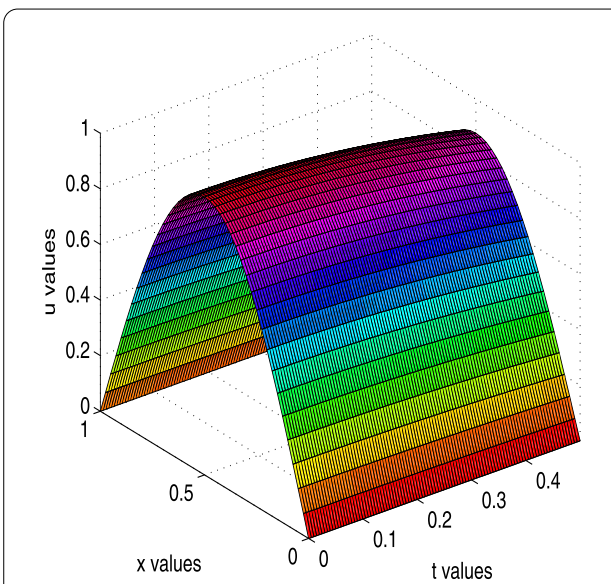

(a) Numerical Solution

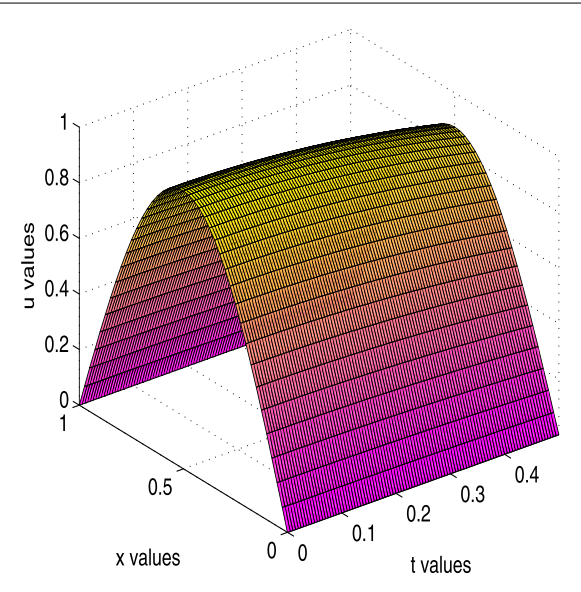

(b) Exact Solution

Figure 1 Example 1: The graph of numerical and exact solutions for $h=0.025$ and $k=0.005$.

Table 2 The absolute errors for the nonhomogenous fourth-order parabolic equation (69), Example 2

\begin{tabular}{lllllllll}
\hline Methods & $\boldsymbol{\lambda}$ & Time steps & & $\boldsymbol{x}=\mathbf{0 . 1}$ & $\boldsymbol{x}=\mathbf{0 . 2}$ & $\boldsymbol{x}=\mathbf{0 . 3}$ & $\boldsymbol{x}=\mathbf{0 . 4}$ & $\boldsymbol{x}=\mathbf{0 . 5}$ \\
\hline Proposed $O\left(k^{2}+h^{4}\right)-$ & 0.5 & 16 & $u$ & $2.09(-11)$ & $2.63(-11)$ & $1.23(-10)$ & $1.93(-10)$ & $2.85(-10)$ \\
method $(28 a)-(28 b)$ & & & $u_{x x}$ & $5.06(-09)$ & $1.54(-08)$ & $6.00(-08)$ & $3.40(-08)$ & $3.87(-08)$ \\
$O\left(k^{2}+h^{4}\right)$-method in $[33]$ & 0.5 & 16 & $u$ & $7.46(-10)$ & $2.91(-10)$ & $8.65(-10)$ & $6.87(-10)$ & $6.98(-10)$ \\
$O\left(k^{4}+h^{4}\right)$-method in [33] & 0.5 & 16 & $u$ & $6.25(-10)$ & $2.22(-10)$ & $4.53(-10)$ & $4.41(-10)$ & $5.03(-10)$ \\
\hline
\end{tabular}

Example 3 We seek the numerical solution of the following homogenous variable coefficient problem $[28,29,33]$ :

$$
\left(\frac{1}{x}+\frac{x^{4}}{120}\right) \frac{\partial^{4} u}{\partial x^{4}}+\frac{\partial^{2} u}{\partial t^{2}}=0, \quad \frac{1}{2}<x<1, t>0 .
$$

The exact solution is

$$
u(x, t)=\left(1+\frac{x^{5}}{120}\right) \sin t
$$

In order to compare the results obtained using our proposed methods with those of the existing methods $[28,29,33]$, we have solved this problem using method $(28 \mathrm{a})-(28 \mathrm{~b})$ with $h=0.05$ and $k=0.000125,0.00025,0.000625$ using 80, 40, and 16 time steps, respectively. The maximum absolute relative errors defined as

$$
\max \left|\frac{U_{l}^{j}-u_{l}^{j}}{U_{l}^{j}}\right|, \quad l=1(1) N
$$

are tabulated in Table 3 at $t=0.01$. Numerical comparison with the existing method of the same accuracy $O\left(k^{2}+h^{4}\right)$ as the proposed method (28a)-(28b) demonstrates the superiority of our proposed methods. The $3 \mathrm{D}$ graphs of the numerical solution vs exact solution are plotted in Figure 2(a) and (b), respectively, for $0.5<x<1$ from $t=0$ to 0.01 . 
Table 3 The Maximum absolute relative errors for Example 3 at $t=0.01$ for various values of $\lambda$ (uniform mesh)

\begin{tabular}{|c|c|c|c|c|c|c|}
\hline $\bar{\lambda}$ & & $\begin{array}{l}\text { Proposed } \\
O\left(k^{2}+h^{4}\right)- \\
\text { method } \\
(28 a)-(28 b)\end{array}$ & $\begin{array}{l}O\left(k^{2}+h^{4}\right)- \\
\text { method } \\
\text { discussed } \\
\text { in [33] }\end{array}$ & $\begin{array}{l}O\left(k^{4}+h^{4}\right)- \\
\text { method } \\
\text { discussed } \\
\text { in [33] }\end{array}$ & $\begin{array}{l}\text { Method } \\
\text { discussed } \\
\text { in }[29]\end{array}$ & $\begin{array}{l}\text { Method } \\
\text { discussed } \\
\text { in [28] }\end{array}$ \\
\hline \multirow[t]{2}{*}{0.05} & $u$ & $6.0176(-12)$ & $5.21(-08)$ & $5.33(-08)$ & $9.90(-08)$ & $1.90(-06)$ \\
\hline & $u_{x x}$ & $2.4614(-12)$ & & & & \\
\hline \multirow[t]{2}{*}{0.1} & u & $4.4223(-12)$ & $1.03(-07)$ & $9.97(-08)$ & $8.10(-08)$ & $7.20(-07)$ \\
\hline & $u_{x x}$ & $3.1911(-12)$ & & & & \\
\hline \multirow[t]{2}{*}{0.25} & u & $5.2459(-12)$ & $3.74(-08)$ & $3.51(-08)$ & $6.90(-08)$ & $4.10(-07)$ \\
\hline & $u_{x x}$ & $4.9774(-12)$ & & & & \\
\hline
\end{tabular}

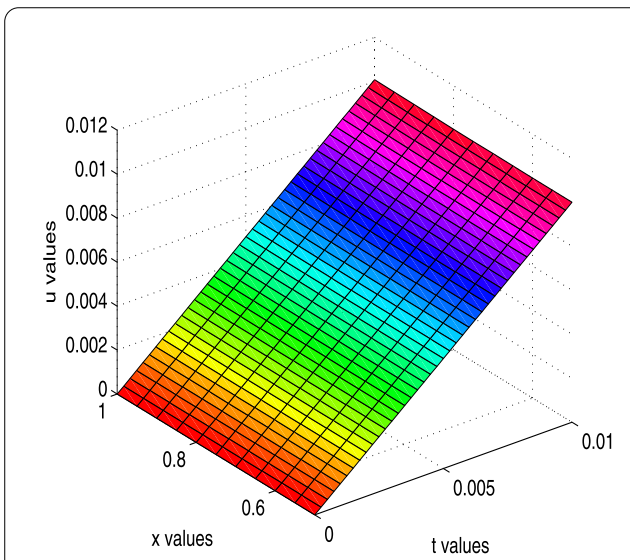

(a) Numerical Solution

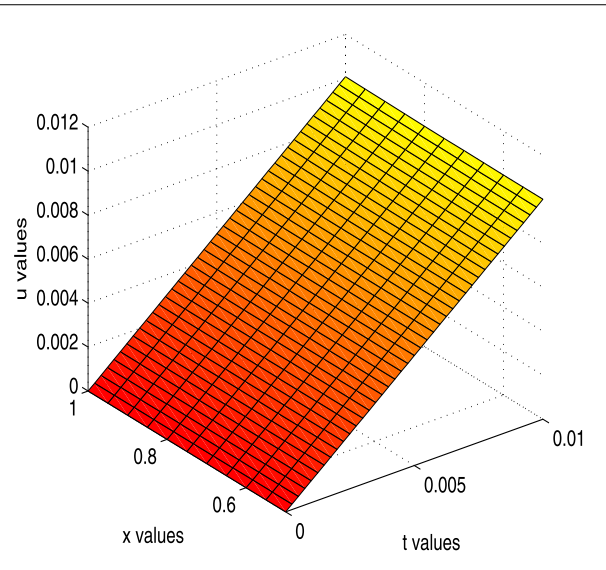

(b) Exact Solution

Figure 2 Example 3: The graph of numerical and exact solutions for $\lambda=0.1, h=0.05$, and $k=0.00025$ for $t=0$ to 0.01 .

Example 4 We consider the singularly perturbed problem of the form

$$
\epsilon \frac{\partial^{4} u}{\partial x^{4}}+\frac{\partial^{2} u}{\partial t^{2}}=f(x, t), \quad 0<\epsilon \ll 1,0<x<1, t>0
$$

The exact solution is

$$
u(x, t)=e^{-\epsilon \pi^{2} t} \sin \pi x .
$$

The maximum absolute errors (MAEs) using methods (28a)-(28b) and (27a)-(27b) are tabulated in Table 4 at $t=1$ for various values of $\epsilon$.

Example 5 We solve numerically the linear singular problem (59) whose exact solution is $u=r^{4} \sin r \sin t$ using difference schemes (61a)-(61b) and (62a)-(62b). The MAEs are tabulated in Table 5 at $t=1$ for $\alpha=1,2$ and $\eta=0.94$. The 3D graphs of numerical solution using method (16a)-(16b) vs exact solution are plotted in Figure 3(a) and (b), respectively for $0<r<1$ from $t=0$ to 1 . 
Table 4 The MAEs for Example 4 at $t=1.0$ for a fixed $\lambda=\left(k / h^{2}\right)=1.6$ (uniform mesh)

\begin{tabular}{|c|c|c|c|c|c|c|c|}
\hline \multirow{2}{*}{\multicolumn{2}{|c|}{$\bar{h}$}} & \multicolumn{3}{|c|}{$O\left(k^{2}+h^{4}\right)$-method (28a)-(28b) } & \multicolumn{3}{|c|}{$O\left(k^{2}+h^{2}\right)$-method (27a)-(27b) } \\
\hline & & \multirow{2}{*}{$\begin{array}{l}\boldsymbol{\epsilon = \mathbf { 0 . 1 }} \\
3.0988(-04)\end{array}$} & \multirow{2}{*}{$\begin{array}{l}\boldsymbol{\epsilon}=\mathbf{0 . 0 1} \\
2.9825(-05)\end{array}$} & \multirow{2}{*}{$\begin{array}{l}\boldsymbol{\epsilon}=\mathbf{0 . 0 0 1} \\
3.5010(-06)\end{array}$} & $=0.1$ & $\epsilon=0.01$ & $\epsilon=0.001$ \\
\hline \multirow[t]{2}{*}{$1 / 8$} & \multirow{2}{*}{$\begin{array}{l}u \\
u_{x x}\end{array}$} & & & & \multirow{2}{*}{$\begin{array}{l}3.2430(-02) \\
3.9514(-01)\end{array}$} & \multirow{2}{*}{$\begin{array}{l}1.0781(-02) \\
1.1690(-01)\end{array}$} & \multirow{2}{*}{$\begin{array}{l}1.1932(-03) \\
1.2865(-02)\end{array}$} \\
\hline & & $2.4409(-03)$ & $3.8680(-04)$ & $4.4212(-05)$ & & & \\
\hline \multirow[t]{2}{*}{$1 / 16$} & \multirow{2}{*}{$\begin{array}{l}u \\
u x x\end{array}$} & \multirow{2}{*}{$\begin{array}{l}1.9387(-05) \\
1.5294(-04)\end{array}$} & \multirow{2}{*}{$\begin{array}{l}1.8905(-06) \\
2.4412(-05)\end{array}$} & $2.2211(-07)$ & \multirow{2}{*}{$\begin{array}{l}8.0703(-03) \\
9.9261(-02)\end{array}$} & \multirow{2}{*}{$\begin{array}{l}2.7648(-03) \\
3.0176(-02)\end{array}$} & \multirow{2}{*}{$\begin{array}{l}3.0660(-04) \\
3.3273(-03)\end{array}$} \\
\hline & & & & $2.7933(-06)$ & & & \\
\hline \multirow[t]{2}{*}{$1 / 32$} & $u$ & $1.2121(-06)$ & $1.1856(-07)$ & $1.3934(-08)$ & $2.0148(-03)$ & $6.9554(-04)$ & $7.7174(-05)$ \\
\hline & $u_{x x}$ & $9.5655(-06)$ & $1.5293(-06)$ & $1.7506(-07)$ & $2.4840(-02)$ & $7.6039(-03)$ & $8.3890(-04)$ \\
\hline
\end{tabular}

Table 5 The MAEs for Example 5 at $t=1.0, \eta=0.94$ (quasi-variable mesh)

\begin{tabular}{|c|c|c|c|c|c|}
\hline \multirow[t]{2}{*}{$N+1$} & & \multicolumn{2}{|c|}{$O\left(k^{2}+k^{2} h_{l}+h_{l}^{3}\right)$-method (16a)-(16b) } & \multicolumn{2}{|c|}{$O\left(k^{2}+h_{l}^{2}\right)$-method (15a)-(15b) } \\
\hline & & $\alpha=1$ & $\alpha=2$ & $\alpha=1$ & $\alpha=2$ \\
\hline \multirow[t]{2}{*}{8} & $u$ & $1.8743(-04)$ & $5.4079(-04)$ & $3.6061(-03)$ & $8.7376(-03)$ \\
\hline & $u_{r r}$ & $1.4353(-03)$ & $8.2498(-03)$ & $8.6067(-02)$ & $7.9741(-02)$ \\
\hline \multirow[t]{2}{*}{16} & $u$ & $1.7017(-05)$ & $4.5019(-05)$ & $6.8621(-04)$ & $1.6912(-03)$ \\
\hline & $u_{r r}$ & $1.5498(-04)$ & $1.3071(-03)$ & $2.6282(-02)$ & $4.7895(-02)$ \\
\hline \multirow[t]{2}{*}{32} & $u$ & $3.1833(-06)$ & $7.4946(-06)$ & $1.2889(-04)$ & $3.5410(-04)$ \\
\hline & $u_{r r}$ & $5.1980(-05)$ & $4.3430(-04)$ & $1.1033(-02)$ & $3.0090(-02)$ \\
\hline
\end{tabular}

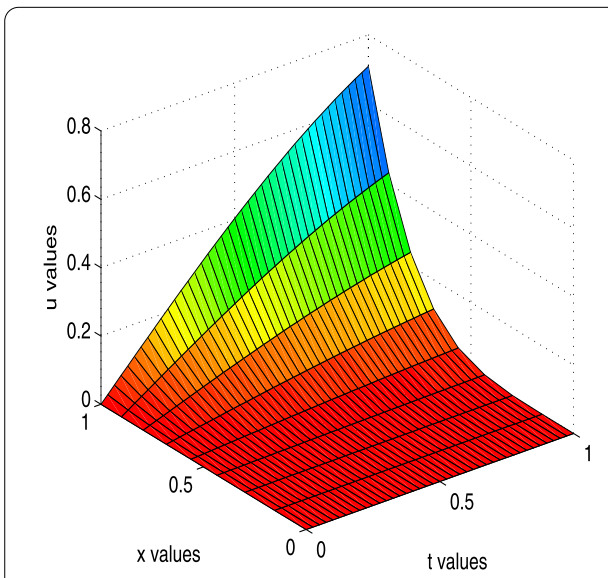

(a) Numerical Solution

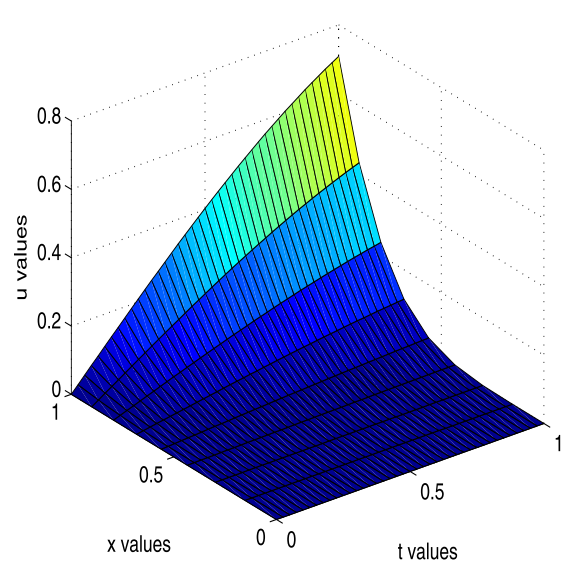

(b) Exact Solution

Figure 3 Example 5: The graph of numerical and exact solutions for $\alpha=1, \eta=0.94$, and $N+1=8$ for $t=0$ to 1.0 .

Example 6 We consider the second-order Benjamin-Ono equation (4) with $q=1$ and $r=-1$. Fu et al. [40] constructed the exact periodic solutions of equation (4) for the above parameters using the Jacobi elliptic function expansion method having the form

$$
u(x, t)=\frac{1}{2} r^{2}-4 l^{2}+6 l^{2} \tanh ^{2}[l(x-r t)] .
$$

To compare our results with the results of Lai and Ma [7], we solve this problem with the difference method (28a)-(28b) with $h=0.1$ and $k=0.01$ taking the same physical constants as in [7]: $l=0.3$ and $r=0.01$ with $[-25,25]$ as the computation domain. The MAEs are tabulated in Table 6 at various time levels $t=5,10,15$, and 20. The 2D graph of the numerical solution vs exact solution is plotted in Figure 4 for $-25<x<25$ at $t=5$. 
Table 6 The MAEs for the second-order Benjamin-Ono equation (4) with $q=1, r=-1$, Example 6 at various time levels for $h=0.1, k=0.01$

\begin{tabular}{|c|c|c|c|}
\hline \multirow[t]{2}{*}{$t$} & \multicolumn{2}{|c|}{$O\left(k^{2}+h^{4}\right)$-method (28a)-(28b) } & \multirow{2}{*}{$\frac{\text { Method discussed in [7] }}{u}$} \\
\hline & $\bar{u}$ & $u_{x x}$ & \\
\hline 5 & $4.2188(-07)$ & $2.4846(-07)$ & $3.3988(-04)$ \\
\hline 10 & $1.8157(-06)$ & $8.2387(-07)$ & $5.2273(-04)$ \\
\hline 15 & $5.5052(-06)$ & $2.4263(-06)$ & $8.2328(-04)$ \\
\hline 20 & $1.4255(-05)$ & $6.2847(-06)$ & $1.2596(-03)$ \\
\hline
\end{tabular}

Figure 4 Example 6: Comparison between numerical and exact solutions of equation (4) at $t=5$ for $h=0.1$ and $k=0.01$.

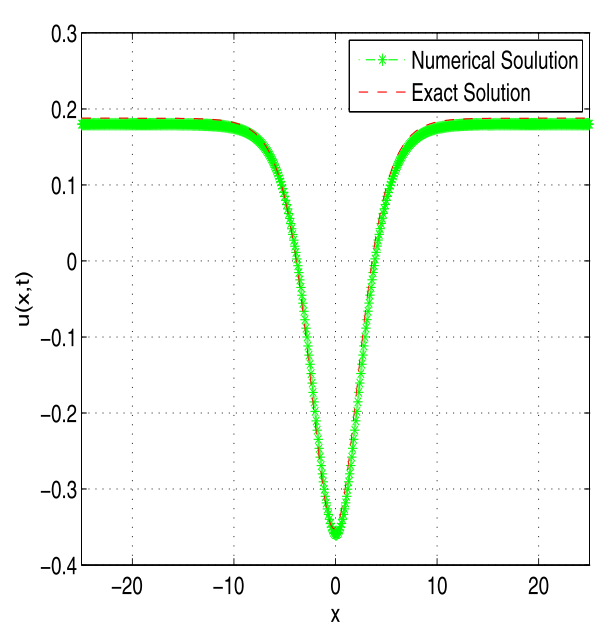

Table 7 The MAEs for the good Boussinesq equation (5), Example 7 at various time levels for a uniform mesh with $k=0.05$

\begin{tabular}{|c|c|c|c|c|c|}
\hline \multirow[t]{2}{*}{$t$} & \multirow[t]{2}{*}{ Parameters } & & \multicolumn{2}{|c|}{$\begin{array}{l}O\left(k^{2}+h^{4}\right) \text {-method } \\
(28 a)-(28 b)\end{array}$} & \multirow{2}{*}{$\begin{array}{l}\text { Collocation method } \\
\text { discussed in [36] } \\
u\end{array}$} \\
\hline & & & $\bar{u}$ & $u_{x x}$ & \\
\hline 0.5 & $h=1 / 40$ & $x_{0}=30$ & $8.1007(-07)$ & $4.4585(-06)$ & $8.2943(-07)$ \\
\hline 1.0 & $h=1 / 60$ & $x_{0}=40$ & $7.6030(-09)$ & $3.1598(-08)$ & $7.3326(-09)$ \\
\hline 1.5 & $h=1 / 80$ & $x_{0}=50$ & $5.8974(-11)$ & $2.0970(-09)$ & $6.4525(-11)$ \\
\hline 2.0 & $h=1 / 100$ & $x_{0}=60$ & $2.9068(-13)$ & $3.2836(-13)$ & $5.2066(-13)$ \\
\hline
\end{tabular}

Example 7 We consider the good Boussinesq equation (5) on the domain $-25 \leq x \leq 25$ with the following exact solution [36]:

$$
u(x, t)=-A \operatorname{sech}^{2}\left[\sqrt{\frac{A}{6}}\left(x-c t+x_{0}\right)\right]-\left(b+\frac{1}{2}\right) .
$$

This exact solution represents a solitary wave with amplitude $A$ located initially at $x=x_{0}$ and moving to the right or left corresponding to the sign of the velocity $c$. If $c$ is positive (negative), then the solitary wave moves to the right (left). For comparison with [36], we first choose the parameters $A, b$, and $c$ similar to [36], that is, $A=0.369, b=-\frac{1}{2}$, and $c=0.868$ for various values of $x_{0}$. We have solved this problem with the method (28a)(28b) presented in this article at various time levels $t=0.5,1.0,1.5$, and 2.0, and MAEs are reported in Table 7 . 
Table 8 The MAEs for Example 8 at $t=1.0, \eta=0.92$ (quasi-variable mesh)

\begin{tabular}{|c|c|c|c|c|c|c|c|}
\hline \multirow[t]{2}{*}{$N+1$} & & \multicolumn{3}{|c|}{$O\left(k^{2}+k^{2} h_{l}+h_{l}^{3}\right)$-method (16a)-(16b) } & \multicolumn{3}{|c|}{$O\left(k^{2}+h_{l}^{2}\right)$-method (15a)-(15b) } \\
\hline & & $\alpha=10$ & $\alpha=\mathbf{2 0}$ & $\alpha=40$ & $\alpha=10$ & $\alpha=\mathbf{2 0}$ & $\alpha=40$ \\
\hline \multirow[t]{2}{*}{8} & $u$ & $3.3667(-05)$ & $7.7683(-05)$ & $1.7212(-02)$ & $2.6072(-04)$ & $3.0874(-04)$ & $2.6133(-02)$ \\
\hline & $u_{x x}$ & $3.3298(-04)$ & $7.6915(-04)$ & $1.7290(-01)$ & $2.3571(-04)$ & $7.0446(-04)$ & $2.5645(-01)$ \\
\hline \multirow[t]{2}{*}{16} & $u$ & $2.1139(-06)$ & $4.7259(-06)$ & $1.0805(-03)$ & $8.6976(-05)$ & $1.0909(-04)$ & $9.5763(-03)$ \\
\hline & $u_{x x}$ & $2.1334(-05)$ & $4.7049(-05)$ & $1.0817(-02)$ & $1.2171(-04)$ & $3.4424(-04)$ & $9.4655(-02)$ \\
\hline \multirow[t]{2}{*}{32} & $u$ & $1.3319(-07)$ & $2.7901(-07)$ & $7.8958(-05)$ & $4.1496(-05)$ & $5.1293(-05)$ & $3.9491(-03)$ \\
\hline & $u_{x x}$ & $1.5345(-06)$ & $2.9686(-06)$ & $7.9178(-04)$ & $5.4398(-05)$ & $1.5469(-04)$ & $3.9160(-02)$ \\
\hline
\end{tabular}

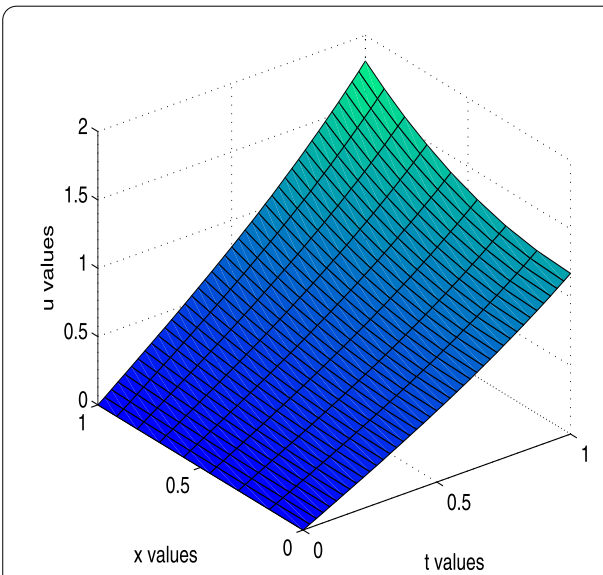

(a) Numerical Solution

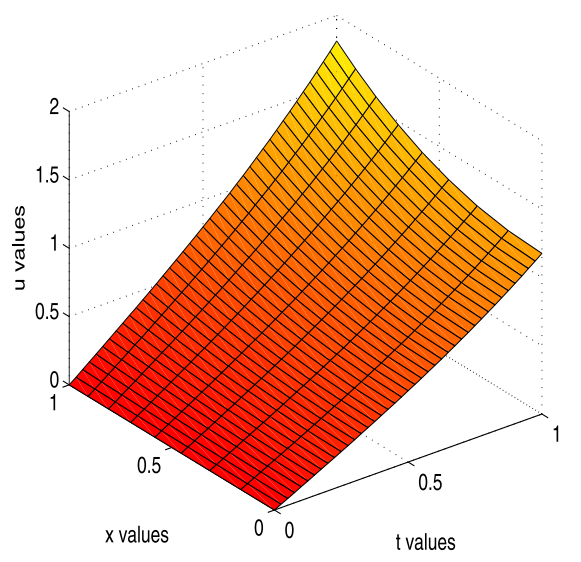

(b) Exact Solution

Figure 5 Example 8: The graph of numerical and exact solutions for $\alpha=20, \eta=0.92$ and $N+1=8$ for $t=0$ to 1.0 .

Example 8 We compute the approximate solution of the following quasi-linear equation:

$$
\left(1+u^{2}+u_{x x}^{2}\right) \frac{\partial^{4} u}{\partial x^{4}}+\frac{\partial^{2} u}{\partial t^{2}}=\alpha u\left(u_{x}-u_{x x}\right)+f(x, t), \quad 0<x<1, t>0 .
$$

The exact solution is

$$
u(x, t)=\cosh x \sinh t .
$$

The MAEs are tabulated in Table 8 at $t=1$ for $\eta=0.92$ and for various values of $\alpha$. The 3D graphs of numerical solution using method (16a)-(16b) vs exact solution are plotted in Figure 5(a) and (b), respectively, for $0<x<1$ from $t=0$ to 1 .

Example 9 We consider the following particular type of fourth-order nonlinear parabolic equations:

$$
\frac{\partial^{4} u}{\partial x^{4}}-2 \frac{\partial^{3} u}{\partial x^{2} \partial t}+\frac{\partial^{2} u}{\partial t^{2}}=\alpha u\left(u_{x x}-u_{t}\right)+g(x, t), \quad 0<x<1, t>0 .
$$

The exact solution is

$$
u=\cosh x \sin t .
$$


Table 9 The MAEs for Example 9 at $t=4.0, \eta=0.92$ (quasi-variable mesh)

\begin{tabular}{|c|c|c|c|c|c|c|c|}
\hline \multirow[t]{2}{*}{$N+1$} & & \multicolumn{3}{|c|}{$O\left(k^{2}+k h_{l}+h_{l}^{3}\right)$-method (37a)-(37b) } & \multicolumn{3}{|c|}{$O\left(k^{2}+h_{l}^{2}\right)$-method (36a)-(36b) } \\
\hline & & $\alpha=1$ & $\alpha=5$ & $\alpha=10$ & $\alpha=1$ & $\alpha=5$ & $\alpha=10$ \\
\hline \multirow[t]{2}{*}{8} & u & $2.5627(-05)$ & $3.5373(-05)$ & $7.1052(-05)$ & $1.1611(-04)$ & $1.1059(-04)$ & $8.9291(-05)$ \\
\hline & $u_{x x}-u_{t}$ & $2.8854(-05)$ & $8.8904(-05)$ & $4.3557(-04)$ & $36(-05)$ & $4.6297(-05)$ & $2.4532(-04)$ \\
\hline \multirow[t]{2}{*}{16} & $u$ & $1.6009(-06)$ & $2.2252(-06)$ & $4.5252(-06)$ & $4.3184(-05)$ & $4.3903(-05)$ & $4.6318(-05)$ \\
\hline & $u_{x x}-u_{t}$ & $1.8900(-06)$ & $5.7189(-06)$ & $2.8222(-05)$ & $2.0745(-06)$ & $8.1349(-06)$ & $3.5144(-05)$ \\
\hline \multirow[t]{2}{*}{32} & $u$ & $9.1931(-08)$ & $1.3617(-07)$ & $2.9842(-07)$ & $2.1699(-05)$ & $2.2259(-05)$ & $2.4254(-05)$ \\
\hline & $u_{x x}-u_{t}$ & $1.4804(-07)$ & $4.2072(-07)$ & $2.0094(-06)$ & $1.7714(-06)$ & $6.1484(-06)$ & $2.7953(-05)$ \\
\hline
\end{tabular}

Table 10 The MAEs for Example 10 at $t=1.0$ for a fixed $\lambda=\left(k / h^{2}\right)=1.6$ (uniform mesh)

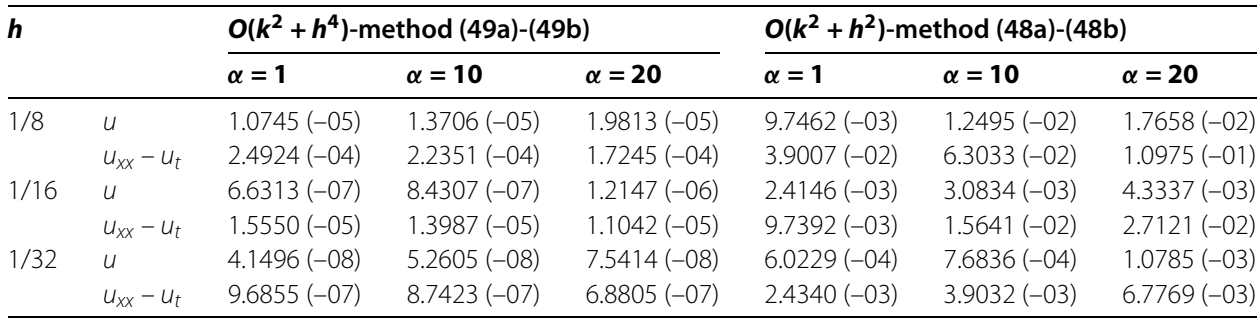

The MAEs using method (36a)-(36b) and (37a)-(37b) are reported in Table 9 for $\eta=0.92$ at $t=4$ using the time step $k=3.2 /(N+1)^{2}$ for various values of $\alpha$.

Example 10 We consider the particular type of fourth-order singular equation of the form:

$$
\frac{\partial^{4} u}{\partial x^{4}}-2 \frac{\partial^{3} u}{\partial x^{2} \partial t}+\frac{\partial^{2} u}{\partial t^{2}}=\frac{\left(u_{x x}-u_{t}\right)}{x}+\frac{\alpha}{x} u+g(x, t), \quad 0<x<1, t>0 .
$$

The exact solution is

$$
u=e^{-t} \sin \pi x \text {. }
$$

The MAEs using method (48a)-(48b) and (49a)-(49b) are reported in Table 10 at $t=1$ for various values of $\alpha$.

\section{Conclusions}

In this paper, we propose finite difference approximations for the fourth-order timedependent parabolic PDEs (1) and (6). The methods were tested on several examples taken from the literature to observe the accuracy and efficiency of the new methods. The results illustrate that the errors in the numerical solution obtained by the current approach are smaller than those obtained by earlier research studies. The main conclusions are:

(i) High-order accuracy: In the case of the uniform mesh, for a fixed value of the mesh ratio parameter $\lambda=\frac{k}{h^{2}}$, the proposed three-level method (28a)-(28b) and two-level method (49a)-(49b) are fourth-order accurate in space. The numerical results for Examples 1, 2, and 3 indicate that the methods produce better results in comparison to the existing methods $[28,29,32-35]$ for the Euler-Bernoulli beam equation. Also, it is seen from Table 6 that the proposed algorithm performs significantly better than the scheme in [7] for the 
second-order Benjamin-Ono equation and is in good agreement with [36] for the nonlinear good Boussinesq equation.

(ii) Compact stencil: The finite difference methods discussed here are based only on three spatial grid points. In each time step, every iteration involves solving a tridiagonal system.

(iii) No Ghost points: The boundary conditions are incorporated in a natural way without the use of any extra nodes or special schemes adjacent to the boundary, thereby eliminating the usual complexity encountered with the difference methods.

(iv) Directly applicable to singular problems: The existing fourth-order implicit difference method of [37] for solving the fourth-order quasi-linear parabolic equation (1) is not directly applicable to problems in polar coordinates and requires a special technique to handle singular points because of the presence of the terms of the form $1 / r_{l-1}$, which give rise to singularity at $l=1$ as $r_{0}=0$. In the present paper, by using off-step nodal points the singularity at $r=0$ is avoided, which enables a direct application of the proposed stable methods for finding the numerical solution of fourth-order parabolic equations with singular coefficients.

(v) Unconditional stability of the two-level method: The two-level implicit methods for the particular type of the fourth-order parabolic PDE (6) are unconditionally stable. Thus, the time step can be considerably large, which is extremely useful when the problem is solved on a long time interval. In Example 6, the maximum absolute errors has been calculated at large time levels $t=5,10,15,20$, and in Example 9, the errors are computed at $t=4$. The accuracy of the schemes is not degraded at large time intervals.

Also, the numerical solution of $u_{x x}$, in case of solution of (1) and the one-dimensional time-dependent Laplacian $u_{x x}-u_{t}$ and in case of solution of (6), which are quite often of interest in various applied problems, are computed as a byproduct of the proposed methods. We are currently working on extension of these methods to solve 2D and 3D fourth-order nonlinear parabolic PDEs. Application of these new methods to some more physical problems in science and engineering will be the content of our further research.

Competing interests

The authors declare that they have no competing interest.

Authors' contributions

RKM discussed the three-level quasi-variable mesh method and partly stability analysis. DK discussed the two-level implicit method, application to singular problems, and partly stability analysis. DK also carried out the computational work. All the authors read and approved the final manuscript.

\section{Author details \\ ${ }^{1}$ Department of Applied Mathematics, Faculty of Mathematics and Computer Science, South Asian University, Akbar Bhawan, Chanakyapuri, New Delhi 110021, India. ${ }^{2}$ Department of Mathematics, Faculty of Mathematical Sciences,} University of Delhi, Delhi, 110007, India.

\section{Acknowledgements}

The authors thank the reviewers for their valuable comments and suggestions, which substantially improved the standard of the paper.

Received: 14 September 2016 Accepted: 29 November 2016 Published online: 13 December 2016

\section{References}

1. Gorman, DJ: Free Vibrations Analysis of Beams and Shafts. Wiley, New York (1975)

2. Meirovitch, L: Principles and Techniques of Vibrations. Prentice Hall, New Jersey (1997)

3. Debnath, L: Nonlinear Partial Differential Equations for Scientists and Engineers. Birkhauser, Boston (1997)

4. Hereman, W, Banerjee, PP, Korpel, A, Assanto, G: Exact solitary wave solutions of non-linear evolution and wave equations using a direct algebraic method. J. Phys. A, Math. Gen. 19, 607-628 (1986) 
5. Xu, ZH, Xian, DQ, Chen, HL: New periodic solitary-wave solutions for the Benjamin-Ono equation. Appl. Math. Comput. 215, 4439-4442 (2010)

6. Soh, CW: Euler-Bernoulli beams from a symmetry standpoint-characterization of equivalent equations. J. Math. Anal. Appl. 345, 387-395 (2008)

7. Lai, H, Ma, C: The lattice Boltzmann model for the second-order Benjamin-Ono equations. J. Stat. Mech., 2010 P04011 (2010)

8. Bratsos, AG: The solution of the Boussinesq equation using the method of lines. Comput. Methods Appl. Mech. Eng. 157, 33-44 (1998)

9. Ismail, MS, Bratsos, AG: A predictor-corrector scheme for the numerical solution of the Boussinesq equation. J. Appl. Math. Comput. 13, 11-27 (2003)

10. Dehghan, M, Salehi, R: A meshless based numerical technique for traveling solitary wave solution of Boussinesa equation. Appl. Math. Model. 36, 1939-1956 (2012)

11. Mohanty, RK, Kaur, D: High accuracy implicit variable mesh methods for numerical study of special types of fourth order non-linear parabolic equations. Appl. Math. Comput. 273, 678-696 (2016)

12. Jain, MK, Jain, RK, Mohanty, RK: A fourth order difference method for the one-dimensional general quasilinear parabolic partial differential equation. Numer. Methods Partial Differ. Equ. 6, 311-319 (1990)

13. Mohanty, RK, Jain, MK, Kumar, D: Single cell finite difference approximations of $O\left(k h^{2}+h^{4}\right)$ for $\partial u / \partial x$ for one space dimensional nonlinear parabolic equation. Numer. Methods Partial Differ. Equ. 16, 408-415 (2000)

14. Mohanty, RK, Karaa, S, Arora, U: An $O\left(k^{2}+k h^{2}+h^{4}\right)$ arithmetic average discretization for the solution of 1-D nonlinear parabolic equations. Numer. Methods Partial Differ. Equ. 23, 640-651 (2007)

15. Mohanty, RK: An implicit high accuracy variable mesh scheme for 1-D non-linear singular parabolic partial differential equations. Appl. Math. Comput. 186, 219-229 (2007)

16. Mohanty, RK, Kumar, R: A new fast algorithm based on half-step discretization for one space dimensional quasilinear hyperbolic equations. Appl. Math. Comput. 244, 624-641 (2014)

17. Mohanty, RK, Gopal, V: High accuracy non-polynomial spline in compression method for one-space dimensional quasi-linear hyperbolic equations with significant first order space derivative term. Appl. Math. Comput. 238, 250-265 (2014)

18. Mohanty, RK, Jha, N, Kumar, R: A new variable mesh method based on non-polynomial spline in compression approximations for 1D quasilinear hyperbolic equations. Adv. Differ. Equ. 2015337 (2015)

19. Talwar, J, Mohanty, RK, Singh, S: A new spline in compression approximation for one space dimensional quasilinear parabolic equations on a variable mesh. Appl. Math. Comput. 260, 82-96 (2015)

20. Talwar, J, Mohanty, RK, Singh, S: A new algorithm based on spline in tension approximation for 1D quasi-linear parabolic equations on a variable mesh. Int. J. Comput. Math. 93, 1771-1786 (2016)

21. Siraj-ul-Islam, Vertnik, R, Šarlen, B: Local radial basis function collocation method along with explicit time stepping for hyperbolic partial differential equations. Appl. Numer. Math. 67, 136-151 (2013)

22. Mohanty, RK, Kaur, D: Numerov type variable mesh approximations for 1D unsteady quasi-linear biharmonic problem: application to Kuramoto-Sivashinsky equation. Numer. Algorithms (2016). doi:10.1007/s1 1075-016-0154-3

23. Conte, SD: A stable implicit finite difference approximation to a fourth order parabolic equation. J. Assoc. Comput. Mech. 4, 18-23 (1957)

24. Crandall, SH: Optimum recurrence formulas for a fourth order parabolic partial differential equation. J. Assoc. Comput. Mach. 4, 467-471 (1957)

25. Evans, DJ: A stable explicit method for the finite difference solution of a fourth order parabolic partial differential equation. Comput. J. 8, 280-287 (1965)

26. Fairweather, G, Gourley, AR: Some stable difference approximations to a fourth order parabolic partial differential equation. Math. Comput. 21, 1-11 (1967)

27. Collatz, L: Hermitian methods for initial value problems in partial differential equations. In: Miller, JJH (ed.) Topics in Numerical Analysis, pp. 41-61. Academic Press, New York (1973)

28. Andrade, C, McKee, S: High accuracy A.D.I methods for fourth-order parabolic equations with variable coefficients. J. Comput. Appl. Math. 3, 11-14 (1977)

29. Twizell, EH, Khaliq, AQM: A difference scheme with high accuracy in time for fourth order parabolic equations. Comput. Methods Appl. Mech. Eng. 41, 91-104 (1983)

30. Evans, DJ, Yousif, WS: A note on solving the fourth-order parabolic equation by the AGE method. Int. J. Comput. Math. 40, 93-97 (1991)

31. Khan, A, Khan, I, Aziz, T: Sextic spline solution for solving a fourth-order parabolic partial differential equation. Int. J. Comput. Math. 82, 871-879 (2005)

32. Caglar, H, Caglar, N: Fifth-degree B-spline solution for a fourth-order parabolic partial differential equations. Appl. Math. Comput. 201, 597-603 (2008)

33. Rashidinia, J, Mohammadi, R: Sextic spline solution of variable coefficient fourth-order parabolic equations. Int. J. Comput. Math. 87, 3443-3454 (2010)

34. Mittal, RC, Jain, RK: B-splines methods with redefined basis functions for solving fourth order parabolic partial differential equations. Appl. Math. Comput. 217, 9741-9755 (2011)

35. Mohammadi, R: Sextic B-spline collocation method for solving Euler-Bernoulli beam models. Appl. Math. Comput. 241, 151-166 (2014)

36. Siddiqi, SS, Arshed, S: Quintic B-spline for the numerical solution of the good Boussinesq equation. J. Egypt. Math. Soc. 22, 209-213 (2014)

37. Mohanty, RK, Evans, DJ: The numerical solution of fourth order mildly qausi-linear parabolic initial boundary value problem of second kind. Int. J. Comput. Math. 80, 1147-1159 (2003)

38. Kelly, CT: Iterative Methods for Linear and Non-linear Equations. SIAM, Philadelphia (1995)

39. Varga, RS: Matrix Iterative Analysis. Springer, New York (2000)

40. Fu, Z, Liu, S, Liu, SD, Zhao, Q: The JEFE method and periodic solutions of two kinds of nonlinear wave equations. Commun. Nonlinear Sci. Numer. Simul. 8, 67-75 (2003) 